\title{
A new bound on the domination number of graphs with minimum degree two
}

\author{
${ }^{1}$ Michael A. Henning;, ${ }^{2}$ Ingo Schiermeyer, and ${ }^{3}$ Anders Yeo \\ ${ }^{1}$ Department of Mathematics \\ University of Johannesburg \\ Auckland Park, 2006 South Africa \\ mahenning@uj.ac.za \\ ${ }^{2}$ Diskrete Mathematik \\ TU Bergakademie Freiberg \\ Institut für Diskrete Mathematik und Algebra \\ 09596 Freiberg Germany \\ schierme@math.tu-freiberg.de \\ ${ }^{3}$ Department of Computer Science \\ Royal Holloway, University of London, Egham \\ Surrey TW20 OEX, UK \\ anders@cs.rhul.ac.uk
}

Submitted: Apr 30, 2009; Accepted: Dec 18, 2010; Published: Jan 5, 2011

Mathematics Subject Classification: 05C69

\begin{abstract}
For a graph $G$, let $\gamma(G)$ denote the domination number of $G$ and let $\delta(G)$ denote the minimum degree among the vertices of $G$. A vertex $x$ is called a bad-cut-vertex of $G$ if $G-x$ contains a component, $C_{x}$, which is an induced 4-cycle and $x$ is adjacent to at least one but at most three vertices on $C_{x}$. A cycle $C$ is called a special-cycle if $C$ is a 5 -cycle in $G$ such that if $u$ and $v$ are consecutive vertices on $C$, then at least one of $u$ and $v$ has degree 2 in $G$. We let bc $(G)$ denote the number of bad-cut-vertices in $G$, and $\operatorname{sc}(G)$ the maximum number of vertex disjoint special-cycles in $G$ that contain no bad-cut-vertices. We say that a graph is $\left(C_{4}, C_{5}\right)$-free if it has no induced 4-cycle or 5-cycle. Bruce Reed [14] showed that if $G$ is a graph of order $n$ with $\delta(G) \geq 3$, then $\gamma(G) \leq 3 n / 8$. In this paper, we relax the minimum degree condition from three to two. Let $G$ be a connected graph of order $n \geq 14$ with $\delta(G) \geq 2$. As
\end{abstract}

${ }^{*}$ Research supported in part by the South African National Research Foundation 
an application of Reed's result, we show that $\gamma(G) \leq \frac{1}{8}(3 n+\operatorname{sc}(G)+\mathrm{bc}(G))$. As a consequence of this result, we have that (i) $\gamma(G) \leq 2 n / 5$; (ii) if $G$ contains no special-cycle and no bad-cut-vertex, then $\gamma(G) \leq 3 n / 8$; (iii) if $G$ is $\left(C_{4}, C_{5}\right)$-free, then $\gamma(G) \leq 3 n / 8$; (iv) if $G$ is 2 -connected and $d_{G}(u)+d_{G}(v) \geq 5$ for every two adjacent vertices $u$ and $v$, then $\gamma(G) \leq 3 n / 8$. All bounds are sharp.

Keywords: bounds, cycles, domination number

AMS subject classification: $05 \mathrm{C69}$

\section{Introduction}

In this paper, we continue the study of domination in graphs. Domination in graphs is now well studied in graph theory. The literature on this subject has been surveyed and detailed in the two books by Haynes, Hedetniemi, and Slater [5, 6].

For notation and graph theory terminology we in general follow [5]. Specifically, let $G=(V, E)$ be a graph with vertex set $V$ of order $n=|V|$ and edge set $E$ of size $m=|E|$, and let $v$ be a vertex in $V$. The open neighborhood of $v$ is the set $N(v)=\{u \in V \mid u v \in E\}$ and the closed neighborhood of $v$ is $N[v]=\{v\} \cup N(v)$. For a set $S$ of vertices, the open neighborhood of $S$ is defined by $N(S)=\cup_{v \in S} N(v)$, and the closed neighborhood of $S$ by $N[S]=N(S) \cup S$. If $X, Y \subseteq V$, then the set $X$ is said to dominate the set $Y$ if $Y \subseteq N[X]$. For a set $S \subseteq V$, the subgraph induced by $S$ is denoted by $G[S]$ while the graph $G-S$ is the graph obtained from $G$ by deleting the vertices in $S$ and all edges incident with $S$. We denote the degree of $v$ in $G$ by $d_{G}(v)$, or simply by $d(v)$ if the graph $G$ is clear from context. The minimum degree among the vertices of $G$ is denoted by $\delta(G)$. A cycle on $n$ vertices is denoted by $C_{n}$.

A dominating set of a graph $G=(V, E)$ is a set $S$ of vertices of $G$ such that every vertex $v \in V$ is either in $S$ or adjacent to a vertex of $S$. (That is, $N[S]=V$.) The domination number of $G$, denoted by $\gamma(G)$, is the minimum cardinality of a dominating set. A dominating set of $G$ of cardinality $\gamma(G)$ is called a $\gamma(G)$-set.

If $G$ does not contain a graph $F$ as an induced subgraph, then we say that $G$ is $F$-free. We say that $G$ is $\left(C_{4}, C_{5}\right)$-free if $G$ is both $C_{4}$-free and $C_{5}$-free; that is, if $G$ has no induced 4-cycle and no induced 5-cycle.

By identifying two vertices $x$ and $y$ in $G$ we mean replacing the vertices $x$ and $y$ by a new vertex $v_{x y}$ and joining $v_{x y}$ to all vertices that were adjacent to $x$ or $y$ in $G$.

\subsection{Reducible Graphs}

In this section, we define two types of reducible graphs. Using these reductions, we define a family $\mathcal{F}_{\leq 13}$ of graphs each of which has order at most 13 .

Definition 1 If there is a path $v_{1} u_{1} u_{2} v_{2}$ on four vertices in a graph $G$ such that $d\left(u_{1}\right)=$ $d\left(u_{2}\right)=2$ in $G$, then we call the graph obtained from $G$ by identifying $v_{1}$ and $v_{2}$ and deleting $\left\{u_{1}, u_{2}\right\}$ a type-1 $G$-reducible graph. 
Definition 2 If there is a path $x_{1} w_{1} w_{2} w_{3} x_{2}$ on five vertices in a graph $G$ such that $d\left(w_{2}\right)=2$ and $N\left(w_{1}\right)=N\left(w_{3}\right)=\left\{x_{1}, x_{2}, w_{2}\right\}$ in $G$, then we call the graph obtained from $G$ by deleting $\left\{w_{1}, w_{2}, w_{3}\right\}$ and adding the edge $x_{1} x_{2}$ if the edge is not already present in $G$ a type-2 $G$-reducible graph.

Definition 3 Let $\mathcal{F}_{4}$ be a set of graphs only containing one element, namely the 4-cycle $C_{4}$. Thus, $\mathcal{F}_{4}=\left\{C_{4}\right\}$. For every $i>4$ with $i \equiv 1(\bmod 3)$, we define the family $\mathcal{F}_{i}$ as follows. A graph $G$ belongs to $\mathcal{F}_{i}$ if and only if $\delta(G) \geq 2$ and there is a type-1 or a type-2 $G$-reducible graph that belongs to $\mathcal{F}_{i-3}$.

Notice that for every $i \geq 4$ with $i \equiv 1(\bmod 3)$, if $G \in \mathcal{F}_{i}$, then $G$ has order $i$. To illustrate Definition 3, consider the graphs $G_{7}, G_{10}$ and $G_{13}$ shown in Figure 1(a), 1(b) and $1(\mathrm{c})$, respectively. Each of these graphs has minimum degree at least two. Note that the 4 -cycle $C_{4}$ is both a type- $1 G_{7}$-reducible graph and a type- $2 G_{7}$-reducible graph. Thus, $G_{7} \in \mathcal{F}_{7}$. The graph $G_{7}$ itself is a type- $1 G_{10}$-reducible graph, and so $G_{10} \in \mathcal{F}_{10}$. The graph $G_{10}$ is a type-2 $G_{13}$-reducible graph, and so $G_{13} \in \mathcal{F}_{13}$.

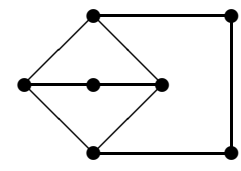

(a) $G_{7}$

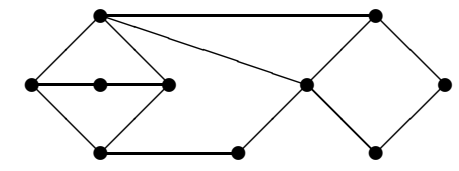

(b) $G_{10}$

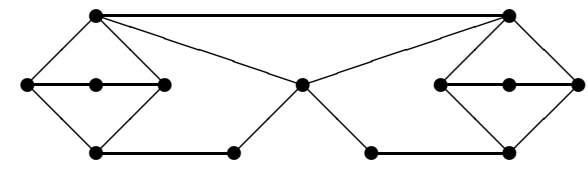

(b) $G_{13}$

Figure 1: The graphs $G_{7}, G_{10}$ and $G_{13}$.

The six graphs in the family $\mathcal{F}_{7}$ are shown in Figure 2. (The graph $G_{7}$ in Figure 1(a) is redrawn as the graph shown in Figure 2(b).)

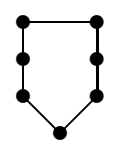

(a)

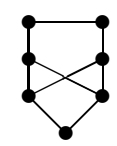

(b)

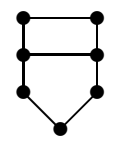

(c)

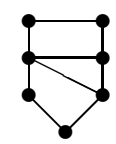

(d)

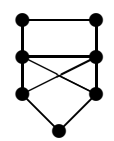

(e)

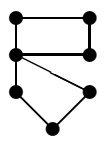

$(f)$

Figure 2: The family $\mathcal{F}_{7}$.

Lemma 1 If $G$ is a graph and $G^{\prime}$ is a type-1 $G$-reducible graph, then $\gamma(G)=\gamma\left(G^{\prime}\right)+1$.

Proof. Let $v_{1} u_{1} u_{2} v_{2}$ be a path in $G$ such that $d\left(u_{1}\right)=d\left(u_{2}\right)=2$ in $G$, and let $G^{\prime}$ be the type-1 $G$-reducible graph obtained from $G$ by identifying $v_{1}$ and $v_{2}$ into one vertex $w$ and deleting $\left\{u_{1}, u_{2}\right\}$. We show first that $\gamma(G) \leq \gamma\left(G^{\prime}\right)+1$. Let $D^{\prime}$ be a $\gamma\left(G^{\prime}\right)$-set. If $w \in D^{\prime}$, let $D=\left(D^{\prime} \backslash\{w\}\right) \cup\left\{v_{1}, v_{2}\right\}$. If $w \notin D^{\prime}$, let $w^{\prime}$ be a vertex in $D^{\prime}$ that dominates $w$ in $G^{\prime}$. Without loss of generality, we may assume that $w^{\prime} \in N_{G}\left(v_{1}\right)$. (Possibly, $w^{\prime}$ is also in the neighborhood of $v_{2}$ in $G$.) In this case, let $D=D^{\prime} \cup\left\{u_{2}\right\}$. In both cases, $D$ is a dominating set of $G$ and $|D|=\left|D^{\prime}\right|+1$. Hence, $\gamma(G) \leq|D|=\gamma\left(G^{\prime}\right)+1$. 
We show next that $\gamma\left(G^{\prime}\right) \leq \gamma(G)-1$. Among all $\gamma(G)$-sets, let $S$ be chosen so that $\left|S \cap\left\{u_{1}, u_{2}\right\}\right|$ is minimum. Then either $\left\{v_{1}, v_{2}\right\} \subseteq S$ or $S \cap\left\{v_{1}, v_{2}\right\}=\emptyset$. If $\left\{v_{1}, v_{2}\right\} \subseteq S$, then let $S^{\prime}=\left(S \backslash\left\{v_{1}, v_{2}\right\}\right) \cup\{w\}$. If $S \cap\left\{v_{1}, v_{2}\right\}=\emptyset$, then we may assume without loss of generality that $S \cap\left\{u_{1}, u_{2}\right\}=\left\{u_{2}\right\}$ (if both $u_{1}$ and $u_{2}$ belong to $S$, replace them by $v_{1}$ and $v_{2}$ to produce a $\gamma(G)$-set that contradicts our choice of $S$ ). In this case, let $S^{\prime}=S \backslash\left\{u_{2}\right\}$. In both cases, $S^{\prime}$ is a dominating set of $G^{\prime}$ and $\left|S^{\prime}\right|=|S|-1$, and so $\gamma\left(G^{\prime}\right) \leq\left|S^{\prime}\right|=\gamma(G)-1$. Consequently, $\gamma(G)=\gamma\left(G^{\prime}\right)+1$.

Lemma 2 If $G$ is a graph and $G^{\prime}$ is a type-2 G-reducible graph, then $\gamma(G)=\gamma\left(G^{\prime}\right)+1$.

Proof. Let $x_{1} w_{1} w_{2} w_{3} x_{2}$ be a path in $G$ such that $d\left(w_{2}\right)=2$ and $N_{G}\left(w_{1}\right)=N_{G}\left(w_{3}\right)=$ $\left\{x_{1}, x_{2}, w_{2}\right\}$, and let $G^{\prime}$ be the type-2 $G$-reducible graph $G^{\prime}=\left(G-\left\{w_{1}, w_{2}, w_{3}\right\}\right) \cup\left\{x_{1} x_{2}\right\}$. We show first that $\gamma(G) \leq \gamma\left(G^{\prime}\right)+1$. Let $D^{\prime}$ be a $\gamma\left(G^{\prime}\right)$-set. If $D^{\prime} \cap\left\{x_{1}, x_{2}\right\}=\emptyset$, let $D=D^{\prime} \cup\left\{w_{2}\right\}$. If $D^{\prime} \cap\left\{x_{1}, x_{2}\right\} \neq \emptyset$, let $D=D^{\prime} \cup\left\{w_{1}\right\}$. Then, $D$ is a dominating set of $G$ and $|D|=\left|D^{\prime}\right|+1$. Hence, $\gamma(G) \leq|D|=\gamma\left(G^{\prime}\right)+1$. We show next that $\gamma\left(G^{\prime}\right) \leq \gamma(G)-1$. Among all $\gamma(G)$-sets, let $S$ be chosen so that $\left|S \cap\left\{w_{1}, w_{2}, w_{3}\right\}\right|$ is minimum. Then, $\left|S \cap\left\{w_{1}, w_{2}, w_{3}\right\}\right|=1$. If $w_{2} \in S$, then $S \cap\left\{w_{1}, w_{3}\right\}=\emptyset$ and we let $S^{\prime}=S \backslash\left\{w_{2}\right\}$. If $w_{2} \notin S$, then we may assume without loss of generality that $\left\{w_{1}, x_{1}\right\} \subseteq S$. Thus, $w_{3} \notin S$ (possibly, $x_{2} \in S$ ). In this case, we let $S^{\prime}=S \backslash\left\{w_{1}\right\}$. In both cases, $S^{\prime}$ is a dominating set of $G^{\prime}$ and $\left|S^{\prime}\right|=|S|-1$, and so $\gamma\left(G^{\prime}\right) \leq\left|S^{\prime}\right|=\gamma(G)-1$. Consequently, $\gamma(G)=\gamma\left(G^{\prime}\right)+1$.

Lemma 3 For every $i \geq 4$ where $i \equiv 1(\bmod 3)$, if $G \in \mathcal{F}_{i}$, then $\gamma(G)=(i+2) / 3$.

Proof. We proceed by induction on $i \geq 4$. When $i=4, G=C_{4}$ and $\gamma(G)=2=(i+2) / 3$. This establishes the base case. Assume, then, that $i \geq 7$ and $i \equiv 1(\bmod 3)$, and that the theorem holds for all $i^{\prime} \geq 4$ where $i^{\prime} \equiv 1(\bmod 3)$ and $i^{\prime}<i$. Let $G \in \mathcal{F}_{i}$. Then there is a graph $G^{\prime}$ in the family $\mathcal{F}_{i-3}$ that is a type-1 $G$-reducible graph or a type-2 $G$-reducible graph. By the induction hypothesis, $\gamma\left(G^{\prime}\right)=(i-1) / 3$. By Lemmas 1 and 2 , $\gamma(G)=\gamma\left(G^{\prime}\right)+1=(i+2) / 3$.

Definition 4 Let $\mathcal{F}_{\leq 13}=\mathcal{F}_{4} \cup \mathcal{F}_{7} \cup \mathcal{F}_{10} \cup \mathcal{F}_{13}$.

We close this section with the following useful properties of graphs in the family $\mathcal{F}_{\leq 13}$.

Lemma 4 Let $G \in \mathcal{F}_{\leq 13}$ have order $n$. Then the following hold:

(a) $n \leq 13$.

(b) $\gamma(G)=(n+2) / 3$.

(c) If $G \in \mathcal{F}_{10} \cup \mathcal{F}_{13}$, then $\gamma(G) \leq 2 n / 5$.

(d) If $G$ contains a triangle, then at most one vertex in this triangle has degree 2 in $G$.

Proof. Statement $(a)$ follows from the fact that each graph in $\mathcal{F}_{i}$ has order $i$. Statement $(b)$ is a consequence of Lemma 3, while Statement $(c)$ is a consequence of Statements $(a)$ and $(b)$. Statement $(d)$ follows from the observation that if there is a triangle 
in $G$ that contains two vertices of degree 2 in $G$, then every type- $1 G$-reducible graph and every type- $2 G$-reducible graph must also contain a triangle that contains two vertices of degree 2 in the resulting graph. Continuing this process, we would reach a contradiction since the 4 -cycle, which is the only graph in $\mathcal{F}_{4}$, contains no triangle.

\section{Known Results}

The decision problem to determine whether the domination number of a graph is at most some given integer is known to be NP-complete. Hence it is of interest to determine upper bounds on the domination number of a graph. In 1989, McCuaig and Shepherd [12] presented the beautiful result that the domination number of a connected graph with minimum degree at least 2 is at most two-fifths its order except for seven exceptional graphs. These seven exceptional graphs are precisely the graphs in the family $\mathcal{F}_{4} \cup \mathcal{F}_{7}$. Hence the McCuaig-Shepherd result can be stated as follows:

Theorem 1 (McCuaig and Shepherd [12]) If $G$ is a connected graph of order $n$ with $\delta(G) \geq 2$ and $G \notin \mathcal{F}_{4} \cup \mathcal{F}_{7}$, then $\gamma(G) \leq 2 n / 5$.

Remark 1. Equality in the bound of Theorem 1 is obtained for infinitely many graphs which are characterized in [12]. We remark that every extremal graph of large order that achieves equality in the bound of Theorem 1 has induced 4-cycles or induced 5-cycles.

Remark 2. We remark that there are infinitely many 2-connected graphs that achieve equality in the bound of Theorem 1. One such family can be constructed as follows: Let $k \geq 2$ be an integer and let $\mathcal{G}_{2 \text { conn }}$ be the family of all graphs that can be obtained from a 2-connected graph $H$ of order $2 k$ that contains a perfect matching $M$ as follows. For each edge $e=u v$ in the matching $M$, duplicate the edge $e$, subdivide one of the duplicated edges twice and subdivide the other duplicated edge once. (Hence each edge $u v$ is deleted from $H$ and replaced by a 5-cycle containing $u$ and $v$ as nonadjacent vertices on the cycle.) Let $G$ denote the resulting graph of order $n=5 k$. Then, $\gamma(G)=2 k=2 n / 5$. A graph in the family $\mathcal{G}_{2 \text { conn }}$ with $k=4$ that is obtained from an 8-cycle $H$ is shown in Figure 3 .

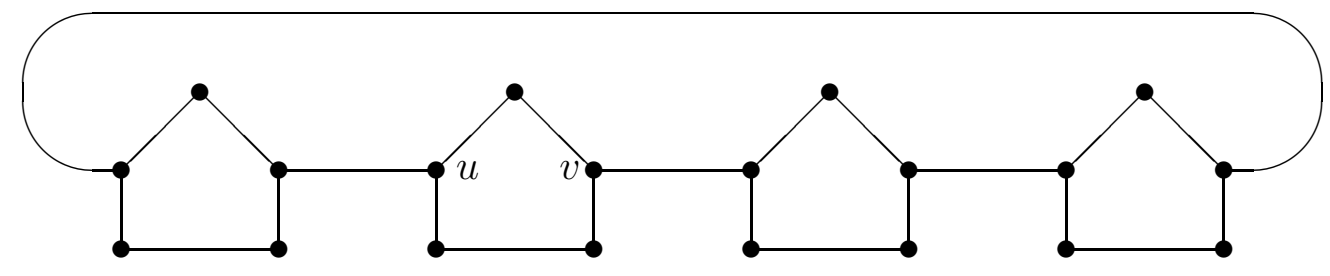

Figure 3: A graph in the family $\mathcal{G}_{2 \text { conn }}$.

The family $\mathcal{G}_{2 \text { conn }}$ we have constructed is a family of 2-connected graphs that achieve equality in the bound of Theorem 1. We remark, however, that every vertex in a graph that belongs to the family $\mathcal{G}_{2 \text { conn }}$ is contained in an induced 5-cycle in that graph. Further every graph in $\mathcal{G}_{2 \text { conn }}$ contains two adjacent degree-2 vertices. 
In 1996, Reed [14] presented the important and useful result that if we restrict the minimum degree to be at least three, then the upper bound in Theorem 1 can be improved from two-fifths its order to three-eights its order.

Theorem 2 (Reed [14]) If $G$ is a graph of order $n$ with $\delta(G) \geq 3$, then $\gamma(G) \leq 3 n / 8$.

The ratio $3 / 8$ in the above theorem is best possible. Gamble gave infinitely many connected graphs of minimum degree at least three with domination number exactly three-eights their order (see $[12,14]$ ). Several authors attempted to improve the $3 / 8$ ratio by restricting the structure of the graph. Kawarabayashi, Plummer, Saito [8] proved that for a 2-edge-connected cubic graph $G$ of girth at least 9 , the $3 / 8$ ratio can be improved to 11/30, while Kostochka and Stodolsky [10] proved that for every connected cubic graph of order at least 10 , the $3 / 8$ ratio can be improved to $4 / 11$. Kostochka and Stodolsky [9] showed that the supremum of $\gamma(G) /|V(G)|$ over connected cubic graphs is at least $8 / 23$, but have no guess what the exact value is. Stodolsky [17] showed that this supremum of $\gamma(G) /|V(G)|$ over 2-connected cubic graphs is at least $9 / 26$.

Molloy and Reed [13] showed that the domination number of a random cubic graph of order $n$ lies between $0.236 n$ and $0.3126 n$ with asymptotic probability 1 . Duckworth and Wormald [1] present an algorithm for finding in a cubic graph of order $n$, drawn uniformly at random, a dominating set of size at most $0.27942 n$ asymptotically almost surely.

Löwenstein and Rautenbach [11] showed that if we relax the minimum degree condition in Reed's Theorem 2 from three to two, but impose a girth condition of girth $g \geq 5$, then the domination number $\gamma$ satisfies $\gamma \leq\left(\frac{1}{3}+\frac{2}{3 g}\right) n$. Recently, Harant and Rautenbach [4] proved the following result.

Theorem 3 (Harant, Rautenbach [4]) If $G$ is a graph of order $n$ with $\delta(G) \geq 2$ that does not contain cycles of length $4,5,7,10$ or 13 , then $\gamma(G) \leq 3 n / 8$.

\section{Main Results}

The result we establish is a fundamental result on the domination number of a graph that cannot be improved in any substantial way in the sense that we establish precisely what structural properties force up the domination number, namely special types of cutvertices (whose removal produces an induced 4-cycle) and special types of 5-cycles. We have several aims in this paper.

Our first aim is to improve the upper bound of McCuaig and Shepherd [12] in Theorem 1 in two instances: First when $G$ is a $\left(C_{4}, C_{5}\right)$-free connected graph with minimum degree at least two. Secondly when $G$ is a 2-connected graph satisfying $d_{G}(u)+d_{G}(v) \geq 5$ for every two adjacent vertices $u$ and $v$. As a byproduct of our results we also obtain a different proof of the McCuaig-Shepherd Theorem 1. Since our proof uses Reed's result, this shows that the beautiful McCuaig-Shepherd result can be deduced from Reed's important result.

Our second aim is to show that the ratio $3 / 8$ in Reed's Theorem 2 holds if we relax the minimum degree condition from three to two, but restrict the structure of the graph 
by forbidding special types of cut-vertices whose removal produces induced 4-cycles and forbidding special types of 5-cycles.

Our third aim is to show that it is unnecessary to forbid cycles of length 7,10 or 13 in the Harant-Rautenbach result, namely Theorem 3, for order $n \geq 14$.

To accomplish these aims, we shall need the concepts of an $X$-dominating set, an $X$ cut-vertex, an $X$-special-cycle, as well as the definition of a family $\mathcal{F}$ of graphs (standing for "forbidden graphs").

\subsection{Restricted Domination}

Definition 5 An $X$-dominating set, abbreviated $X-D S$, in a graph $G$ is a dominating set $S$ of vertices of $G$ such that $X \subseteq S$. The $X$-domination number of $G$, denoted by $\gamma(G ; X)$, is the minimum cardinality of an $X-D S$. An $X-D S$ of $G$ of cardinality $\gamma(G ; X)$ is called a $\gamma(G ; X)$-set.

Note that the $\emptyset$-dominating sets in $G$ are precisely the dominating sets in $G$. Thus, $\gamma(G)=\gamma(G ; \emptyset)$. We remark that the concept of an $X$-DS was introduced by Sanchis in [15] who coined the term restricted domination in graphs since among all dominating sets, we restrict our attention to those that contain the specified subset, $X$, of vertices. The concept of restricted domination in graphs was studied further in $[2,7,16]$ and elsewhere.

\subsection{Bad-Cut-Vertices}

Definition 6 Let $G$ be a graph and let $X \subseteq V(G)$. A vertex $x \in V(G)$ is called an $X$-cut-vertex of $G$ if $x \notin X$ and $G-x$ contains a component, $C_{x}$, which is an induced 4-cycle and which does not contain any vertices from $X$. Furthermore $x$ is adjacent to at least one but at most three vertices on $C_{x}$. Let $\mathrm{bc}(G ; X)$ (standing for 'bad cut-vertex') denote the number of $X$-cut-vertices in $G$. When $X=\emptyset$, we call an $X$-cut-vertex of $G$ a bad-cut-vertex of $G$ and we denote $\mathrm{bc}(G ; X)$ simply by $\mathrm{bc}(G)$. Thus, $\operatorname{bc}(G)$ is the number of bad-cut-vertices in $G$.

\subsection{Special Cycles}

We define a vertex in a graph $G$ as small if has degree 2 in $G$ and large if it has degree more than 2 in $G$.

Definition 7 Let $G$ be a graph and let $X \subseteq V(G)$. We say that a cycle $C$ in a graph $G$ is an $X$-special-cycle if $C$ is a 5 -cycle in $G$ which does not contain any vertices from $X$ and such that if $u$ and $v$ are consecutive vertices on $C$, then at least one of $u$ and $v$ has degree 2 in $G$. Note that if $C$ is an $X$-special-cycle in $G$, then $C$ contains at most two large vertices and these two vertices are not consecutive vertices of $C$ although they may be adjacent in $G$. Let $\operatorname{sc}(G ; X)$ (standing for 'special cycle') denote the maximum number of vertex disjoint $X$-special-cycles in $G$ that contain no $X$-cut-vertex. When $X=\emptyset$, we 
call an $X$-special-cycle of $G$ a special cycle of $G$ and we denote $\operatorname{sc}(G ; X)$ simply by $\operatorname{sc}(G)$. Thus, $\operatorname{sc}(G)$ is the maximum number of vertex disjoint special cycles in $G$ that contain no bad-cut-vertex.

\subsection{The Function $\psi$}

Definition 8 Let $G$ be a graph and let $X \subseteq V(G)$. Let $\delta_{1}(G ; X)$ denote the number of degree-1 vertices in $G$ that do not belong to $X$.

For any graph $G$, and for a subset $X$ of vertices in $G$, let

$$
\psi(G ; X)=\frac{1}{8}\left(3|V(G)|+5|X|+\operatorname{sc}(G ; X)+\mathrm{bc}(G ; X)+2 \delta_{1}(G ; X)\right) .
$$

To illustrate the definition of $\psi(G ; X)$, let $G$ be the graph shown in Figure 4 and let $X=\{x\}$. The vertex labelled $v$ is a $X$-cut-vertex of $G$. As $|V(G)|=13,|X|=1$, $\operatorname{sc}(G ; X)=1, \operatorname{bc}(G ; X)=1$, and $\delta_{1}(G ; X)=1$, we have $\psi(G ; X)=6$. Note that for this graph $G, \gamma(G ; X)=6=\psi(G ; X)$.

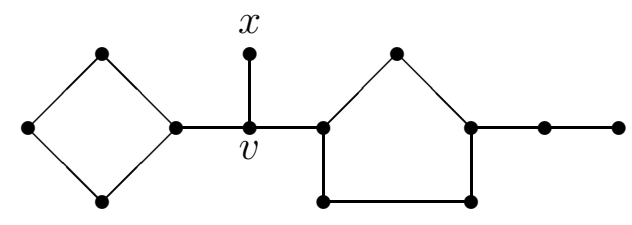

Figure 4: A graph $G$.

The following observations will prove useful.

Observation 1 Let $G$ be a graph with $\delta(G) \geq 1$ and let $X \subseteq V(G)$. Then the following hold:

(a) If $\delta(G) \geq 2$, then $\delta_{1}(G ; X)=0$.

(b) $\operatorname{sc}(G)+\mathrm{bc}(G) \leq|V(G)| / 5$.

(c) If $G$ is $\left(C_{4}, C_{5}\right)$-free, then $\operatorname{sc}(G ; X)=0$.

(d) If $G$ is $C_{4}$-free, then $\operatorname{bc}(G ; X)=0$.

(e) If $G$ is 2-connected and $|V(G)| \neq 5$, then $\operatorname{bc}(G ; X)=0$.

(f) If $d_{G}(u)+d_{G}(v) \geq 5$ for every two adjacent vertices $u$ and $v$, then $\operatorname{sc}(G)=0$.

\subsection{The Graph Family $\mathcal{F}$}

In this section we define a family $\mathcal{F}$ of graphs (standing for "forbidden graphs"). We remark that there are 28076 non-isomorphic graphs in the family $\mathcal{F}_{\leq 13}$ defined in Section 1.1. Of these 28076 graphs in $\mathcal{F}_{\leq 13}$ which we generated by a computer program, 41 of them possess bad-cut-vertices. We now define a family $\mathcal{F}$ of (forbidden) graphs. Let

$$
\mathcal{F}=\left\{G \in \mathcal{F}_{\leq 13} \mid \operatorname{bc}(G)=0\right\}
$$


that is, $\mathcal{F}$ consists of the 28035 non-isomorphic graphs in the family $\mathcal{F}_{\leq 13}$ that do not have a bad-cut-vertex. The following properties of graphs in the family $\mathcal{F}$ will prove to be useful.

Lemma 5 Let $G \in \mathcal{F}$ and let $\{u, v\} \subset V(G)$. Then $G$ has the following properties:

(a) $\gamma(G-v)=\gamma(G)-1$.

(b) There is a $\gamma(G)$-set containing $v$.

(c) There is a $\gamma(G)$-set containing both $u$ and $v$.

(d) If $u v \notin E(G)$ and $G+u v \notin \mathcal{F}$, then $\gamma(G+u v)=\gamma(G)-1$.

Proof. We only have a computer proof of (a), (c) and (d). By Property (a), every $\gamma(G-$ $v$ )-set can be extended to a $\gamma(G)$-set by adding to it the vertex $v$, implying Property (b).

\subsection{Statement of Main Result}

We are now in a position to present our main result.

Theorem 4 Let $G$ be a connected graph and let $X \subseteq V(G)$. If $d_{G}(x) \geq 1$ for all $x \in$ $V(G) \backslash X$, then either $X=\emptyset$ and $G \in \mathcal{F}$ or $\gamma(G ; X) \leq \psi(G ; X)$.

Setting $X=\emptyset$ in Theorem 4, we have the following consequence of Theorem 4 and Observation 1(a). This key result we state as a theorem due to its importance.

Theorem 5 If $G$ is a connected graph with $\delta(G) \geq 2$, then $G \in \mathcal{F}$ or

$$
\gamma(G) \leq \frac{1}{8}(3|V(G)|+\operatorname{sc}(G)+\operatorname{bc}(G)) .
$$

As a consequence of Theorem 5, we have the following results.

Corollary 1 If $G$ is a connected graph of order $n$ with $\delta(G) \geq 2$ that contains no special cycle and no bad-cut-vertex, then either $G \in \mathcal{F}$ or $\gamma(G) \leq 3 n / 8$.

Corollary 2 If $G$ is a connected graph of order $n \geq 14$ with $\delta(G) \geq 2$ that contains no special cycle and no bad-cut-vertex, then $\gamma(G) \leq 3 n / 8$.

Note that if $G$ is a graph with $\delta(G) \geq 3$, then $G$ contains no special cycle and no bad-cut-vertex and $G \notin \mathcal{F}$. Hence Theorem 2 due to Reed is an immediate consequence of Corollary 1. We also remark that Theorem 1 due to McCuaig and Shepherd [12] is an immediate consequence of Theorem 5, Lemma 4(c) and Observation 1(b).

There are several other consequences of Theorem 5 which we list below. Corollary 3 follows from Theorem 5 and Observations 1(c) and 1(d). Corollary 4 follows from Lemma 4(a) and Corollary 3. Corollary 5 follows from Theorem 5 and Observations 1(e) and $1(\mathrm{f})$. 
Corollary 3 If $G \notin \mathcal{F}$ is a $\left(C_{4}, C_{5}\right)$-free connected graph of order $n$ with $\delta(G) \geq 2$, then $\gamma(G) \leq 3 n / 8$.

Corollary 4 If $G$ is a $\left(C_{4}, C_{5}\right)$-free connected graph of order $n \geq 14$ with $\delta(G) \geq 2$, then $\gamma(G) \leq 3 n / 8$.

Corollary 5 If $G$ is a 2-connected graph of order $n \geq 14$ and $d_{G}(u)+d_{G}(v) \geq 5$ for every two adjacent vertices $u$ and $v$, then $\gamma(G) \leq 3 n / 8$.

We remark that there are several graphs in the family $\mathcal{F}$ that are $\left(C_{4}, C_{5}\right)$-free. The simplest such examples are the cycles $C_{n}$, where $n \in\{7,10,13\}$. An example of a $\left(C_{4}, C_{5}\right)$ free graph in the family $\mathcal{F}$ that is not a cycle is shown in Figure 5.

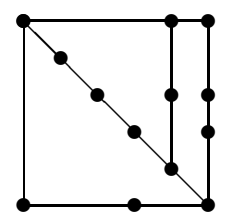

Figure 5: $\mathrm{A}\left(C_{4}, C_{5}\right)$-free graph in the family $\mathcal{F}$.

\subsection{Sharpness of Corollary 3 and Corollary 4}

To illustrate the sharpness of Corollary 3 and Corollary 4, we define a cycle-unit to be a graph that is isomorphic to a cycle $C_{8}$ and a key-unit to be a graph that is isomorphic to a key $L_{7,1}$, where $L_{7,1}$ is the graph of order 8 obtained from a cycle $C_{7}$ by attaching a pendant edge to a vertex in the cycle. In a cycle-unit, we select an arbitrary vertex $v$ and the two vertices at distance three from $v$ in the unit and we call these three vertices the attachers of the cycle-unit, while in a key-unit we call the vertex of degree one the attacher of the key-unit.

Let $\mathcal{G}$ denote the family of all graphs $G$ that are obtained from the disjoint union of $\ell \geq 2$ cycle-unit or key-unit by adding $\ell-1$ edges in such a way that $G$ is connected and every added edge joins two attachers. Note that an attacher may be incident with any number of link edges, including the possibility of zero. Every edge of $G$ joining two attachers we call a link edge of $G$ and we call the resulting two attachers link vertices of $G$. A graph in the family $\mathcal{G}$ with four cycle-units and two key-units is shown in Figure 6 with the link vertices indicated by the large darkened vertices. Note that every link edge of $G$ is a bridge of $G$ and that the attacher in every key-unit of $G$ is the link vertex of the key-unit, while every cycle-unit of $G$ has either one, two or three link vertices. We remark that it is possible that an attacher is incident with no link edge and is therefore not a link vertex. Thus every link vertex is an attacher, but every attacher is not necessarily a link vertex. Every graph in the family $\mathcal{G}$ is a $\left(C_{4}, C_{5}\right)$-free connected graph with minimum degree two and domination number exactly three-eights its order. 


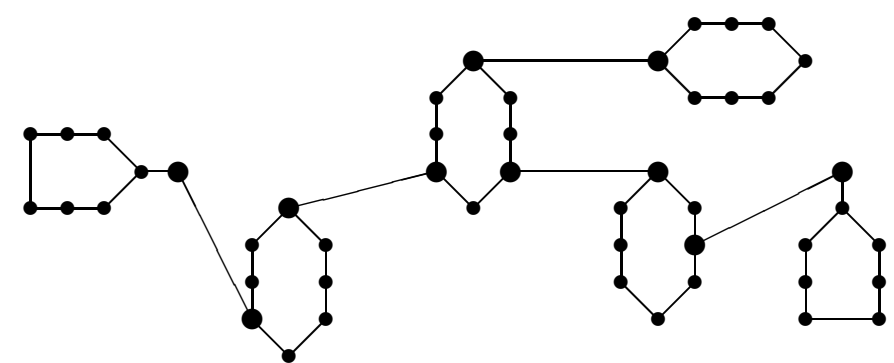

Figure 6: A graph $G$ in the family $\mathcal{G}$.

\subsection{Sharpness of Corollary 5}

To illustrate the sharpness of Corollary 5 , let $k \geq 2$ be an integer and let $\mathcal{H}$ be the family of all graphs that can be obtained from a 2-connected graph $F$ of order $2 k$ that contains a perfect matching $M$ as follows. Replace each edge $e=u v$ in the matching $M$ by an 8-cycle uavbcdefu with two added edges, namely be and $c f$. Let $H$ denote the resulting 2-connected graph of order $n=8 k$. Then, $\gamma(H)=3 k=3 n / 8$ and the set of degree-2 vertices in $H$ form an independent set. A graph in the family $\mathcal{H}$ with $k=4$ that is obtained from an 8-cycle $F$ is shown in Figure 7.

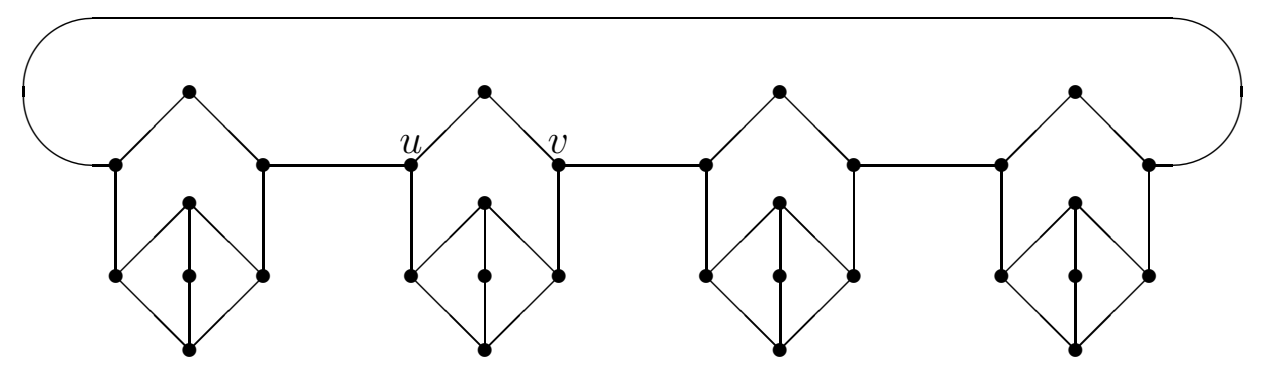

Figure 7: A graph in the family $\mathcal{H}$.

We remark that Corollary 5 can be restated as follows: If $G$ is a 2-connected graph of order $n \geq 14$ such that the set of degree-2 vertices in $G$ form an independent set, then $\gamma(G) \leq 3 n / 8$.

\section{Proof of Theorem 4}

Recall the statement of Theorem 4 .

Theorem 4. Let $G$ be a connected graph and let $X \subseteq V(G)$. If $d_{G}(x) \geq 1$ for all $x \in V(G) \backslash X$, then either $X=\emptyset$ and $G \in \mathcal{F}$ or $\gamma(G ; X) \leq \psi(G ; X)$.

Since our detailed proof of Theorem 4 is very technical, we provide here only a summary of the main ideas of the proof. A detailed proof of Theorem 4 is provided in the appendix. 
Summary of the proof of Theorem 4 . We proceed by induction on the lexicographic sequence $(|V(G)|-|X|,|V(G)|)$. For notational convenience, for a graph $G$ and a subset $X \subseteq V(G)$ and a graph $G^{\prime}$ and a subset $X^{\prime} \subseteq V\left(G^{\prime}\right)$, we denote the sequence $(|V(G)|-$ $|X|,|V(G)|)$ by $s(G)$ and the sequence $\left(\left|V\left(G^{\prime}\right)\right|-\left|X^{\prime}\right|,\left|V\left(G^{\prime}\right)\right|\right)$ by $s\left(G^{\prime}\right)$.

When $|V(G)|-|X|=0$, we have that $V(G)=X$, and $\gamma(G ; X)=|X|=\psi(G ; X)$. This establishes the base case. Assume, then, that $|V(G)|-|X| \geq 1$ and that for all connected graphs $G^{\prime}$ and subsets $X^{\prime} \subseteq V\left(G^{\prime}\right)$ for which $d_{G^{\prime}}(x) \geq 1$ for every $x \in V\left(G^{\prime}\right) \backslash X^{\prime}$ that have lexicographic sequence $s\left(G^{\prime}\right)$ smaller than $s$, we have either $X^{\prime}=\emptyset$ and $G^{\prime} \in \mathcal{F}$ or $\gamma\left(G^{\prime} ; X^{\prime}\right) \leq \psi\left(G^{\prime} ; X^{\prime}\right)$. Let $G$ be a connected graph and let $X$ be a subset of vertices in $G$ such that $d_{G}(x) \geq 1$ for all $x \in V(G) \backslash X$ and with lexicographic sequence $s(G)=s$. We proceed further with a series of claims that we may assume the graph $G$ to satisfy if the result does not hold.

We first show that $|V(G)| \geq 3$ and that $\delta_{1}(G ; X)=0$. From this we deduce that $d_{G}(x) \geq 2$ for all $x \in V(G) \backslash X$. Thus if $X=\emptyset$, then $\delta(G) \geq 2$. We then establish that there is no $X$-cut-vertex in $G$ and no $X$-special-cycle in $G$; that is, bc $(G ; X)=0$ and $\operatorname{sc}(G ; X)=0$. We show next that no vertex of $X$ is a cut-vertex of $G$.

Next we consider the set $S$ of all vertices of $G$ of degree 2 which do not belong to $X$; that is, $S=\left\{x \in V(G) \backslash X \mid d_{G}(x)=2\right\}$. We prove various properties of the set $S$. First we show that $S \neq \emptyset$. Thereafter we prove that there is no path of length 2 in $G[S]$. Next we establish that there is no path of length 1 in $G[S]$. Thus, $S$ is an independent set in $G$. Hence a neighbor of a vertex of $S$ in $G$ is a large vertex or belongs to $X$. We show then that $N(S) \cap X=\emptyset$, and so both neighbors of a vertex of $S$ in $G$ are large vertices and do not belong to $X$; that is, if $v \in S$ and $u \in N(v)$, then $u \notin X$ and $d_{G}(u) \geq 3$. Thereafter we establish that no two vertices of $S$ belong to a common 4-cycle that contains a vertex of degree at least 4 in $G$. We then prove that no two vertices of $S$ belong to a common 4-cycle. Using our assumptions to date, we establish that the set $S$ is a packing in $G$; that is, every two distinct vertices in $S$ are at distance at least 3 apart in $G$.

We then consider a vertex $u \in S$ and let $N(u)=\{v, w\}$. By our earlier observations, we note that $\{v, w\} \cap X=\emptyset$ and every vertex within distance 2 from $u$ in $G$ that does not belong to $X$ has degree at least 3 in $G$. Let $G^{\prime}=G-N[u]$ and let $X^{\prime}=X$. Thus, $\left|V\left(G^{\prime}\right)\right|=|V(G)|-3$ and $\left|X^{\prime}\right|=|X|$. Let $G_{1}$ be a component of $G^{\prime}$ and let $X_{1}=X \cap V\left(G_{1}\right)$. We then show that if $\gamma\left(G_{1} ; X_{1}\right)>\psi\left(G_{1} ; X_{1}\right)$, then $G^{\prime}=G_{1}$. Further, we show that $\gamma\left(G^{\prime} ; X^{\prime}\right) \leq \psi\left(G^{\prime} ; X^{\prime}\right)$ and $\operatorname{sc}\left(G^{\prime} ; X^{\prime}\right)=0$. We show next that the degree-2 vertex $u$ can be chosen so that $\mathrm{bc}\left(G^{\prime} ; X^{\prime}\right)=0$. Thus since every vertex at distance 2 from $u$ in $G$ that does not belong to $X$ has degree at least 3 in $G$, we have that $d_{G^{\prime}}(x) \geq 1$ for all $x \in V\left(G^{\prime}\right) \backslash X^{\prime}$. Finally, we show that $\delta_{1}\left(G^{\prime} ; X^{\prime}\right)=0$. Thus, $\psi\left(G^{\prime} ; X^{\prime}\right)=\psi(G ; X)-9 / 8<$ $\psi(G ; X)-1$. Every $\gamma\left(G^{\prime} ; X^{\prime}\right)$-DS can be extended to a $X$-DS of $G$ by adding to it the vertex $u$, and so $\gamma(G ; X) \leq \gamma\left(G^{\prime} ; X^{\prime}\right)+1<\psi(G ; X)$. This completes the proof of Theorem 4. 


\section{References}

[1] W. Duckworth, N. C. Wormald, Minimum independent dominating sets of random cubic graphs. Random Structures Algorithms 21 (2002), 147-161.

[2] W. Goddard and M. A. Henning, Restricted domination parameters in Graphs. J. Combin. Optimization 13 (2007), 353-363.

[3] J. Harant, A. Pruchnewski, and M. Voigt, On dominating sets and independent sets of graphs. Combin. Probab. Comput. 8 (1999), 547-553.

[4] J. Harant and D. Rautenbach, Domination in bipartite graphs. Discrete Math. 309 (2009), 113-122.

[5] T. W. Haynes, S. T. Hedetniemi, and P. J. Slater (eds.), Fundamentals of Domination in Graphs, Marcel Dekker, Inc. New York, 1998.

[6] T. W. Haynes, S. T. Hedetniemi, and P. J. Slater (eds.), Domination in Graphs: Advanced Topics, Marcel Dekker, Inc. New York, 1998.

[7] M. A. Henning, Restricted domination in graphs. Discrete Math. 254 (2002), 175189.

[8] K. Kawarabayashi, M. D. Plummer, and A. Saito, Domination in a graph with a 2-factor. J. Graph Theory 52 (2006), 1-6.

[9] A. V. Kostochka and B. Y. Stodolsky, On domination in connected cubic graphs. Discrete Math. 304 (2005), 45-50.

[10] A. V. Kostochka and B. Y. Stodolsky, An upper bound on the domination number of $n$-vertex connected cubic graphs. Discrete Math. 309 (2009), 1142-1162.

[11] C. Löwenstein and D. Rautenbach, Domination in graphs with minimum degree at least two and large girth. Graphs Combin. 24 (2008), 37-46.

[12] W. McCuaig and B. Shepherd, Domination in graphs with minimum degree two. J. Graph Theory 13 (1989), 749-762.

[13] M. Molloy and B. Reed, The dominating number of a random cubic graph. Random Structures Algorithms 7 (1995), 209-221.

[14] B. A. Reed, Paths, stars and the number three. Combin. Probab. Comput. 5 (1996), $277-295$.

[15] L. A. Sanchis, Bounds related to domination in graphs with minimum degree two. J. Graph Theory 25 (1997), 139-152.

[16] L. A. Sanchis, Relating the size of a connected graph to its total and restricted domination numbers. Discrete Math. 283 (2004), 205-216.

[17] B. Y. Stodolsky, On domination in 2-connected cubic graphs. Electron. J. Combin. 15 (2008), no. 1, Note 38, 5 pp. 


\section{Detailed Proof of Theorem 4}

We begin with a preliminary observation. Let $G$ be an arbitrary graph. By attaching $a G_{8}$-unit to a specified vertex $v$ of $G$, we mean adding a (disjoint) copy of the graph $G_{8}$ of Figure 8 and identifying any one of its vertices that is in a triangle with $v$.

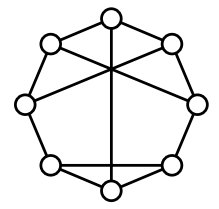

Figure 8: A cubic graph $G_{8}$ with domination number 3

We will use the following observation in the proof of Theorem 4.

Observation 2 If $G^{\prime}$ is obtained from a graph $G$ by attaching a $G_{8}$-unit to a vertex $v$, then there exists a $\gamma\left(G^{\prime}\right)$-set that contains $v$ and two other vertices in the $G_{8}$-unit.

To prove Theorem 4, we proceed by induction on the lexicographic sequence $(|V(G)|-$ $|X|,|V(G)|)$. For notational convenience, for a graph $G$ and a subset $X \subseteq V(G)$ and a graph $G^{\prime}$ and a subset $X^{\prime} \subseteq V\left(G^{\prime}\right)$, we denote the sequence $(|V(G)|-|X|,|V(G)|)$ by $s(G)$ and the sequence $\left(\left|V\left(G^{\prime}\right)\right|-\left|X^{\prime}\right|,\left|V\left(G^{\prime}\right)\right|\right)$ by $s\left(G^{\prime}\right)$.

When $|V(G)|-|X|=0$, we have that $V(G)=X$, and $\gamma(G ; X)=|X|=\psi(G ; X)$. This establishes the base case. Assume, then, that $|V(G)|-|X| \geq 1$ and that for all connected graphs $G^{\prime}$ and subsets $X^{\prime} \subseteq V\left(G^{\prime}\right)$ for which $d_{G^{\prime}}(x) \geq 1$ for every $x \in V\left(G^{\prime}\right) \backslash X^{\prime}$ that have lexicographic sequence $s\left(G^{\prime}\right)$ smaller than $s$, we have either $X^{\prime}=\emptyset$ and $G^{\prime} \in \mathcal{F}$ or $\gamma\left(G^{\prime} ; X^{\prime}\right) \leq \psi\left(G^{\prime} ; X^{\prime}\right)$. Let $G$ be a connected graph and let $X$ be a subset of vertices in $G$ such that $d_{G}(x) \geq 1$ for all $x \in V(G) \backslash X$ and with lexicographic sequence $s(G)=s$. We proceed further with a series of claims that we may assume the graph $G$ to satisfy if the result does not hold.

Claim A $|V(G)| \geq 3$.

Proof. Suppose $G=K_{2}$. Then, $|V(G)|=2$ and $\operatorname{sc}(G ; X)=\operatorname{bc}(G ; X)=0$. Since $|V(G)|-|X| \geq 1$, we have that $X=\emptyset$ or $|X|=1$. If $X=\emptyset$, then $\delta_{1}(G ; X)=2$ and $\gamma(G ; X)=1<6 / 8+2 / 4=3|V(G)| / 8+\delta_{1}(G ; X) / 4=\psi(G ; X)$. If $|X|=1$, then $\delta_{1}(G ; X)=1$ and $\gamma(G ; X)=1=6 / 8+1 / 4=\psi(G ; X)$. Hence if $G=K_{2}$, then the desired result holds. Hence we may assume that $|V(G)| \geq 3$.

Claim B $\delta_{1}(G ; X)=0$.

Proof. Suppose that $\delta_{1}(G ; X) \geq 1$. Then there is a degree- 1 vertex $v$ in $G$ such that $v \notin X$. Let $u$ be the neighbor of $v$. By Claim A, $d_{G}(u) \geq 2$. Let $G^{\prime}=G-v$ and let $X^{\prime}=X \cup\{u\}$. Then, $\left|V\left(G^{\prime}\right)\right|=|V(G)|-1$ and $\left|X^{\prime}\right|=|X|$ (if $u \in X$ ) or $\left|X^{\prime}\right|=$ $|X|+1$ (if $u \notin X)$, and so $\left|V\left(G^{\prime}\right)\right|-\left|X^{\prime}\right|<|V(G)|-|X|$. Further we note that $\left|X^{\prime}\right| \geq$ 
1. Hence by induction there is a $X^{\prime}$-DS $D^{\prime}$ in $G^{\prime}$ such that $\left|D^{\prime}\right| \leq \psi\left(G^{\prime} ; X^{\prime}\right)$. Since $u \in D^{\prime}$, the set $D^{\prime}$ is also a $X$-DS in $G$, and so $\gamma(G ; X) \leq\left|D^{\prime}\right| \leq \psi\left(G^{\prime} ; X^{\prime}\right)$. As $\operatorname{sc}\left(G^{\prime} ; X^{\prime}\right) \leq \operatorname{sc}(G ; X), \operatorname{bc}\left(G^{\prime} ; X^{\prime}\right) \leq \operatorname{bc}(G ; X)$ and $\delta_{1}\left(G^{\prime} ; X^{\prime}\right)=\delta_{1}(G ; X)-1$, we note that $\psi\left(G^{\prime} ; X^{\prime}\right) \leq 3(|V(G)|-1) / 8+5(|X|+1 \mid) / 8+\operatorname{sc}(G ; X) / 8+\mathrm{bc}(G ; X) / 8+\left(\delta_{1}(G ; X)-1\right) / 4=$ $\psi(G ; X)-3 / 8+5 / 8-1 / 4=\psi(G ; X)$. Thus, $\gamma(G ; X) \leq \psi\left(G^{\prime} ; X^{\prime}\right) \leq \psi(G ; X)$, as desired.

As an immediate consequence of Claim $\mathrm{B}$, we have the following claim.

Claim C $d_{G}(x) \geq 2$ for all $x \in V(G) \backslash X$.

Note that if $X=\emptyset$, then Claim C implies that $\delta(G) \geq 2$.

Claim D bc $(G ; X)=0$.

Proof. Suppose bc $(G ; X)>0$. Let $x$ be a $X$-cut-vertex in $G$ and let $H$ be a component in $G-x$ that is an induced 4-cycle not containing any vertices from $X$. By definition, $x \notin X$. Let $u$ be a neighbor of $x$ in $V(H)$, and let $v$ be the vertex at distance 2 from $u$ in the 4-cycle $H$. Let $G^{\prime}=G-V(H)$ and let $X^{\prime}=X \cup\{x\}$. In particular, we note that $\left|X^{\prime}\right| \geq 1$. Since $G$ is connected, so too is $G^{\prime}$. By induction there is a $X^{\prime}$-DS $D^{\prime}$ in $G^{\prime}$ such that $\left|D^{\prime}\right| \leq \psi\left(G^{\prime} ; X^{\prime}\right)$. Let $D=D^{\prime} \cup\{v\}$. Then, $D$ is a $X$-DS in $G$, and so $\gamma(G ; X) \leq|D|=\left|D^{\prime}\right|+1 \leq \psi\left(G^{\prime} ; X^{\prime}\right)+1$. As $\left|V\left(G^{\prime}\right)\right|=|V(G)|-4,\left|X^{\prime}\right|=|X|+1$, $\operatorname{sc}\left(G^{\prime} ; X^{\prime}\right)=\operatorname{sc}(G ; X), \operatorname{bc}\left(G^{\prime} ; X^{\prime}\right)=\mathrm{bc}(G ; X)-1$, and $\delta_{1}\left(G^{\prime} ; X^{\prime}\right)=\delta_{1}(G ; X)=0$, we note that $\psi\left(G^{\prime} ; X^{\prime}\right)=[\psi(G ; X)-12 / 8+5 / 8-1 / 8]=\psi(G ; X)-1$. Thus, $\gamma(G ; X) \leq|D| \leq$ $\psi\left(G^{\prime} ; X^{\prime}\right)+1=\psi(G ; X)$, as desired.

Claim E $\operatorname{sc}(G ; X)=0$.

Proof. Suppose $\operatorname{sc}(G ; X)>0$. Let $C: v_{1} v_{2} v_{3} v_{4} v_{5} v_{1}$ be an $X$-special-cycle in $G$. Renaming vertices if necessary, we may assume that $v_{2}, v_{4}, v_{5}$ are small vertices of $G$. Possibly, $v_{1}$ or $v_{3}$ or both $v_{1}$ and $v_{3}$ are large vertices, and possibly $v_{1} v_{3}$ is an edge of $G$. Let $G^{\prime}=G\left[V(G) \backslash\left\{v_{2}, v_{4}, v_{5}\right\}\right] \cup\left\{v_{1} v_{3}\right\}$ and let $X^{\prime}=X \cup\left\{v_{1}, v_{3}\right\}$. In particular, we note that $\left|X^{\prime}\right| \geq 2$. Since $G$ is connected, so too is $G^{\prime}$. By induction there is a $X^{\prime}$-DS $D^{\prime}$ in $G^{\prime}$ such that $\left|D^{\prime}\right| \leq \psi\left(G^{\prime} ; X^{\prime}\right)$. As $\left|V\left(G^{\prime}\right)\right|=|V(G)|-3,\left|X^{\prime}\right|=|X|+2, \operatorname{sc}\left(G^{\prime} ; X^{\prime}\right)=$ $\operatorname{sc}(G ; X)-1, \operatorname{bc}\left(G^{\prime} ; X^{\prime}\right)=\operatorname{bc}(G ; X)=0$, and $\delta_{1}\left(G^{\prime} ; X^{\prime}\right)=\delta_{1}(G ; X)=0$, we note that $\psi\left(G^{\prime} ; X^{\prime}\right)=\psi(G ; X)-9 / 8+10 / 8-1 / 8=\psi(G ; X)$. Since $D^{\prime}$ is an $X$-DS of $G$, we have that $\gamma(G ; X) \leq\left|D^{\prime}\right| \leq \psi(G ; X)$, as desired.

Claim $\mathbf{F}$ No vertex of $X$ is a cut-vertex of $G$.

Proof. Suppose that $x \in X$ is a cut-vertex of $G$. Let $H_{1}, H_{2}, \ldots, H_{\ell}, \ell \geq 2$, be the components of $G-x$. For $i=1,2, \ldots, \ell$, let $G_{i}=G\left[V\left(H_{i}\right) \cup\{x\}\right]$. Hence, $G$ is obtained from the disjoint union $G_{1} \cup G_{2} \cup \cdots \cup G_{\ell}$ by identifying the vertex $x$ from each graph $G_{i}$ into one common vertex. For $i=1,2, \ldots, \ell$, let $X_{i}=X \cap V\left(G_{i}\right)$, and note that $x \in X_{i}$ and that each $G_{i}$ is a connected graph such that $d_{G_{i}}(x) \geq 2$ for all $x \in V\left(G_{i}\right) \backslash X_{i}$. Applying the inductive hypothesis to $G_{i}$, there is a $X_{i}$-DS $D_{i}$ in $G_{i}$ such that $\left|D_{i}\right| \leq \psi\left(G_{i} ; X_{i}\right)$. Since $x \in X_{i}$, we have that $x \in D_{i}$ for each $i=1,2, \ldots, \ell$. Let 


$$
D=\bigcup_{i=1}^{\ell} D_{i}
$$

Then, $D$ is a $X$-DS in $G$, and so

$$
\gamma(G ; X) \leq|D|=\left(\sum_{i=1}^{\ell}\left|D_{i}\right|\right)-(\ell-1) \leq\left(\sum_{i=1}^{\ell} \psi\left(G_{i} ; X_{i}\right)\right)-(\ell-1)
$$

As

$$
\begin{aligned}
|V(G)| & =\sum_{i=1}^{\ell}\left|V\left(G_{i}\right)\right|-(\ell-1), \\
|X| & =\sum_{i=1}^{\ell}\left|X_{i}\right|-(\ell-1), \\
\operatorname{bc}(G ; X)+\operatorname{sc}(G ; X) & =\sum_{i=1}^{\ell}\left(\operatorname{bc}\left(G_{i} ; X_{i}\right)+\operatorname{sc}\left(G_{i} ; X_{i}\right)\right), \\
\delta_{1}(G ; X) & =\sum_{i=1}^{\ell} \delta_{1}\left(G_{i} ; X_{i}\right),
\end{aligned}
$$

we have that

$$
\sum_{i=1}^{\ell} \psi\left(G_{i} ; X_{i}\right) \leq \psi(G ; X)+\frac{3}{8}(\ell-1)+\frac{5}{8}(\ell-1)=\psi(G ; X)+(\ell-1) .
$$

Hence, by Equation (1) and Equation (2), we have that $\gamma(G ; X) \leq \psi(G ; X)$, as desired.

Let $S$ be the set of all vertices of $G$ of degree 2 which do not belong to $X$; that is, $S=\left\{x \in V(G) \backslash X \mid d_{G}(x)=2\right\}$.

\section{Claim G $S \neq \emptyset$.}

Proof. Suppose $S=\emptyset$. Then, $d_{G}(x) \geq 3$ for every vertex $x \in V(G) \backslash X$. Let $G^{\prime}$ be obtained from $G$ by attaching a $G_{8}$-unit to every vertex of $X$ in $G$. Then, $\delta\left(G^{\prime}\right) \geq 3$. Note that $\left|V\left(G^{\prime}\right)\right|=|V(G)|+7|X|, \operatorname{sc}\left(G^{\prime} ; X^{\prime}\right)=\operatorname{sc}(G ; X)=0, \operatorname{bc}\left(G^{\prime} ; X^{\prime}\right)=\operatorname{bc}(G ; X)=0$, and $\delta_{1}\left(G^{\prime} ; X^{\prime}\right)=\delta_{1}(G ; X)=0$. By Reed's Theorem $2, \gamma\left(G^{\prime}\right) \leq 3\left|V\left(G^{\prime}\right)\right| / 8=3(|V(G)|+$ $7|X|) / 8$. By Observation 2, there exists a $\gamma\left(G^{\prime}\right)$-set $D^{\prime}$ that contains $X$ and two vertices in each $G_{8}$-unit that do not belong to $X$. Let $D$ be the restriction of $D^{\prime}$ to $G$; that is, $D=D^{\prime} \cap V(G)$. Then, $D$ is an $X$-DS of $G$. Hence, $\gamma(G ; X) \leq|D|=\left|D^{\prime}\right|-2|X|=$ $\gamma\left(G^{\prime}\right)-2|X| \leq 3(|V(G)|+7|X|) / 8-2|X|=3|V(G)| / 8+5|X| / 8=\psi(G ; X)$, as desired.

Claim $\mathbf{H}$ There is no path of length 2 in $G[S]$.

Proof. Suppose that there is a path, $u_{1} u_{2} u_{3}$, of length 2 in $G[S]$. Let $N\left(u_{1}\right)=\left\{u_{2}, v_{1}\right\}$ and $N\left(u_{3}\right)=\left\{u_{2}, v_{3}\right\}$. We proceed further with the following subclaim. 
Subclaim H1 $v_{1} \neq v_{3}$.

Proof. Suppose $v_{1}=v_{3}$. If $d_{G}\left(v_{1}\right)=2$, then $G=C_{4}$ and $v_{1} \in X$. In this case, $\gamma(G ; X) \leq\left|\left\{u_{1}, v_{1}\right\}\right|=2<12 / 8+5 / 8=\psi(G ; X)$, as desired. Hence we may assume that $d_{G}\left(v_{1}\right) \geq 3$. Since $v_{1}$ is a cut-vertex of $G, v_{1} \notin X$ by Claim F.

Suppose $d_{G}\left(v_{1}\right)=3$. Let $w_{1}$ be the neighbor of $v_{1}$ different from $u_{1}$ and $u_{3}$. Then $w_{1} \in X$, for otherwise $w_{1}$ would be an $X$-cut-vertex, contradicting our assumption that $\mathrm{bc}(G ; X)=0$. If $d_{G}\left(w_{1}\right) \geq 2$, then $w_{1}$ would be a cut-vertex of $G$, contradicting Claim F. Hence, $d_{G}\left(w_{1}\right)=1$, and so $|V(G)|=5$ and $|X|=1$. Then $\left\{u_{2}, w_{1}\right\}$ is an $X$-DS in $G$, and so $\gamma(G ; X) \leq 2<20 / 8=3|V(G)| / 8+5|X| / 8=\psi(G ; X)$, as desired. Hence we may assume that $d_{G}\left(v_{1}\right) \geq 4$.

Let $G^{\prime}=G-\left\{u_{1}, u_{2}, u_{3}\right\}$ and let $X^{\prime}=X$. In particular, we note that $d_{G^{\prime}}\left(v_{1}\right) \geq 2$. Since $G$ is connected, so too is $G^{\prime}$. Thus we can apply the inductive hypothesis to $G^{\prime}$.

Suppose there is a $X^{\prime}$-DS $D^{\prime}$ in $G^{\prime}$ such that $\left|D^{\prime}\right| \leq \psi\left(G^{\prime} ; X^{\prime}\right)$. Then, $D^{\prime} \cup\left\{u_{2}\right\}$ is a $X$ DS in $G$, and so $\gamma(G ; X) \leq\left|D^{\prime}\right|+1 \leq \psi\left(G^{\prime} ; X^{\prime}\right)+1$. As $\left|V\left(G^{\prime}\right)\right|=|V(G)|-3,\left|X^{\prime}\right|=|X|$, $\operatorname{sc}\left(G^{\prime} ; X^{\prime}\right)+\mathrm{bc}\left(G^{\prime} ; X^{\prime}\right) \leq 1=\operatorname{sc}(G ; X)+\mathrm{bc}(G ; X)+1$, and $\delta_{1}\left(G^{\prime} ; X^{\prime}\right)=\delta_{1}(G ; X)=0$, we have that $\gamma(G ; X) \leq \psi\left(G^{\prime} ; X^{\prime}\right)+1 \leq[\psi(G ; X)-9 / 8+1 / 8]+1=\psi(G ; X)$, as desired. Hence we may assume that there is no $X^{\prime}$-DS $D^{\prime}$ in $G^{\prime}$ such that $\left|D^{\prime}\right| \leq \psi\left(G^{\prime} ; X^{\prime}\right)$.

By induction, $X^{\prime}=X=\emptyset$ and $G^{\prime} \in \mathcal{F}$. Let $\left|V\left(G^{\prime}\right)\right|=n^{\prime}$. Then, $n^{\prime} \in\{4,7,10,13\}$. By Lemma $4(\mathrm{~b}), \gamma\left(G^{\prime}\right)=\left(n^{\prime}+2\right) / 3=(|V(G)|-1) / 3$. Any dominating set of $G^{\prime}$ can be extended to a dominating set of $G$ by adding to it the vertex $u_{2}$, and so $\gamma(G ; X)=$ $\gamma(G) \leq \gamma\left(G^{\prime}\right)+1=(|V(G)|+2) / 3$. If $n^{\prime}=13$, then $|V(G)|=16$ and $\gamma(G ; X)=\gamma(G) \leq$ $6=3|V(G)| / 8=\psi(G ; X)$. If $n^{\prime} \in\{4,7,10\}$, then $G \in \mathcal{F}$.

By Subclaim H1, $v_{1} \neq v_{3}$. Note that it is possible that $v_{1} v_{3} \in E(G)$. Suppose that $N_{G}\left(v_{1}\right)=\left\{u_{1}, v_{3}\right\}$ and $v_{1} \notin X$. Then, $C: v_{1} u_{1} u_{2} u_{3} v_{3} v_{1}$ is an induced cycle in $G$ with at most one large vertex, namely the vertex $v_{3}$. Since $\operatorname{sc}(G ; X)=0$, the cycle $C$ is not a $X$-special-cycle in $G$, implying that $v_{3} \in X$. Let $G^{\prime}=G-\left\{u_{1}, u_{2}, u_{3}, v_{1}\right\}$ and let $X^{\prime}=X$. Since $v_{3} \in X^{\prime}$, we note that $\left|X^{\prime}\right| \geq 1$. Applying the inductive hypothesis to $G^{\prime}$, there is a $X^{\prime}$-DS $D^{\prime}$ in $G^{\prime}$ such that $\left|D^{\prime}\right| \leq \psi\left(G^{\prime} ; X^{\prime}\right)$. The set $D^{\prime} \cup\left\{u_{1}\right\}$ is a $X$-DS in $G$, and so $\gamma(G ; X) \leq\left|D^{\prime}\right|+1 \leq \psi\left(G^{\prime} ; X^{\prime}\right)+1$. As $\left|V\left(G^{\prime}\right)\right|=|V(G)|-4,\left|X^{\prime}\right|=|X|$, $\operatorname{sc}\left(G^{\prime} ; X^{\prime}\right)=\operatorname{sc}(G ; X)=0, \operatorname{bc}\left(G^{\prime} ; X^{\prime}\right)=\operatorname{bc}(G ; X)=0$, and $\delta_{1}\left(G^{\prime} ; X^{\prime}\right)=\delta_{1}(G ; X)=0$, we have that $\gamma(G ; X) \leq \psi\left(G^{\prime} ; X^{\prime}\right)+1 \leq[\psi(G ; X)-12 / 8]+1<\psi(G ; X)$, as desired. Hence we may assume that if $N_{G}\left(v_{1}\right)=\left\{u_{1}, v_{3}\right\}$, then $v_{1} \in X$. Similarly, we may assume that if $N_{G}\left(v_{3}\right)=\left\{u_{3}, v_{1}\right\}$, then $v_{3} \in X$.

Let $G^{\prime}=\left(G-\left\{u_{1}, u_{2}, u_{3}\right\}\right) \cup\left\{v_{1} v_{3}\right\}$ and let $X^{\prime}=X$. If $d_{G^{\prime}}\left(v_{1}\right)=1$, then, $N_{G}\left(v_{1}\right)=$ $\left\{u_{1}, v_{3}\right\}$, and so, by assumption, $v_{1} \in X$. Hence, $d_{G^{\prime}}\left(v_{1}\right) \geq 2$ or $v_{1} \in X$. Similarly, $d_{G^{\prime}}\left(v_{3}\right) \geq 2$ or $v_{3} \in X$. Thus, $d_{G^{\prime}}(x) \geq 2$ for all $x \in V\left(G^{\prime}\right) \backslash X^{\prime}$. Since $G$ is connected, so too is $G^{\prime}$. Hence we can apply the inductive hypothesis to $G^{\prime}$.

Suppose there is a $X^{\prime}$-DS $D^{\prime}$ in $G^{\prime}$ such that $\left|D^{\prime}\right| \leq \psi\left(G^{\prime} ; X^{\prime}\right)$. If $v_{1} \in D^{\prime}$, let $D=$ $D^{\prime} \cup\left\{u_{3}\right\}$. If $v_{1} \notin D^{\prime}$ and $v_{3} \in D^{\prime}$, let $D=D^{\prime} \cup\left\{u_{1}\right\}$. If $\left\{v_{1}, v_{3}\right\} \cap D^{\prime}=\emptyset$, let $D=D^{\prime} \cup\left\{u_{2}\right\}$. In all cases, $D$ is a $X$-DS of $G$, and so $\gamma(G ; X) \leq|D|=\left|D^{\prime}\right|+1 \leq \psi\left(G^{\prime} ; X^{\prime}\right)+1$. As $\left|V\left(G^{\prime}\right)\right|=|V(G)|-3,\left|X^{\prime}\right|=|X|, \operatorname{sc}\left(G^{\prime} ; X^{\prime}\right)+\mathrm{bc}\left(G^{\prime} ; X^{\prime}\right) \leq 1=\operatorname{sc}(G ; X)+\mathrm{bc}(G ; X)+1$, and $\delta_{1}\left(G^{\prime} ; X^{\prime}\right)=\delta_{1}(G ; X)=0$, we have that $\gamma(G ; X) \leq \psi\left(G^{\prime} ; X^{\prime}\right)+1 \leq[\psi(G ; X)-9 / 8+$ 
$1 / 8]+1=\psi(G ; X)$, as desired. Hence we may assume that there is no $X^{\prime}$-DS $D^{\prime}$ in $G^{\prime}$ such that $\left|D^{\prime}\right| \leq \psi\left(G^{\prime} ; X^{\prime}\right)$.

By induction, $X^{\prime}=X=\emptyset$ and $G^{\prime} \in \mathcal{F}$. Let $\left|V\left(G^{\prime}\right)\right|=n^{\prime}$. Then, $n^{\prime} \in\{4,7,10,13\}$. By Lemma $4(\mathrm{~b}), \gamma\left(G^{\prime}\right)=\left(n^{\prime}+2\right) / 3=(|V(G)|-1) / 3$. Any dominating set of $G^{\prime}$ can be extended to a dominating set of $G$ by adding one vertex from the set $\left\{u_{1}, u_{2}, u_{3}\right\}$, and so $\gamma(G) \leq \gamma\left(G^{\prime}\right)+1=(|V(G)|+2) / 3$. If $n^{\prime}=13$, then $|V(G)|=16$ and $\gamma(G ; X)=\gamma(G) \leq$ $6 \leq 3|V(G)| / 8=\psi(G ; X)$. If $n^{\prime} \in\{4,7,10\}$, then $G \in \mathcal{F}$.

Claim I There is no path of length 1 in $G[S]$.

Proof. Suppose that there is a path, $u_{1} u_{2}$, of length 1 in $G[S]$. Let $N\left(u_{1}\right)=\left\{u_{2}, v_{1}\right\}$ and $N\left(u_{2}\right)=\left\{u_{2}, v_{2}\right\}$. We proceed further with the following subclaim.

Subclaim I1 $v_{1} \neq v_{2}$.

Proof. Suppose $v_{1}=v_{2}$. If $d_{G}\left(v_{1}\right)=2$, then $G=K_{3}$ and $v_{1} \in X$. In this case, $\gamma(G ; X)=\left|\left\{v_{1}\right\}\right|=1<9 / 8+5 / 8=\psi(G ; X)$, as desired. Hence we may assume that $d_{G}\left(v_{1}\right) \geq 3$. Since $v_{1}$ is a cut-vertex of $G, v_{1} \notin X$ by Claim F. Let $G^{\prime}=G-\left\{u_{1}, u_{2}\right\}$ and let $X^{\prime}=X \cup\left\{v_{1}\right\}$. By the inductive hypothesis, there is a $X^{\prime}$-DS $D^{\prime}$ in $G^{\prime}$ such that $\left|D^{\prime}\right| \leq \psi\left(G^{\prime} ; X^{\prime}\right)$. Since $D^{\prime}$ is a $X$-DS in $G$, we have that $\gamma(G ; X) \leq\left|D^{\prime}\right| \leq \psi\left(G^{\prime} ; X^{\prime}\right)$. As $\left|V\left(G^{\prime}\right)\right|=|V(G)|-2,\left|X^{\prime}\right|=|X|+1, \operatorname{sc}\left(G^{\prime} ; X^{\prime}\right)=\operatorname{sc}(G ; X)=0, \operatorname{bc}\left(G^{\prime} ; X^{\prime}\right)=$ $\mathrm{bc}(G ; X)=0$, and $\delta_{1}\left(G^{\prime} ; X^{\prime}\right)=\delta_{1}(G ; X)=0$, we have that $\gamma(G ; X) \leq \psi\left(G^{\prime} ; X^{\prime}\right)=$ $\psi(G ; X)-6 / 8+5 / 8<\psi(G ; X)$, as desired.

By Subclaim I1, $v_{1} \neq v_{2}$. (Possibly, $v_{1} v_{2} \in E(G)$.) Let $G^{\prime}$ be obtained from $G$ by identifying $v_{1}$ and $v_{2}$ into one vertex $w$ and by deleting $\left\{u_{1}, u_{2}\right\}$. Since $G$ is connected, so too is $G^{\prime}$. Note that by identifying $v_{1}$ and $v_{2}$ we cannot generate a vertex of degree 1 as $\operatorname{sc}(G ; X)=0$. If $v_{1}$ or $v_{2}$ belongs to $X$, then let $X^{\prime}=(X \cup\{w\}) \backslash\left\{v_{1}, v_{2}\right\}$. If $\left\{v_{1}, v_{2}\right\} \cap X=\emptyset$, let $X^{\prime}=X$. In both cases, we note that $\left|X^{\prime}\right| \leq|X|$.

By Claim $\mathrm{H}$, for $i \in\{1,2\}, d_{G}\left(v_{i}\right) \geq 3$ or $v_{i} \in X$. Hence if $d_{G^{\prime}}(w)=0$, then $G \in\left\{P_{4}, C_{4}\right\}$ and $X=\left\{v_{1}, v_{2}\right\}$. In this case, $\gamma(G ; X)=2<22 / 8=\psi(G ; X)$. Hence we may assume that $d_{G^{\prime}}(w) \geq 1$. Let $w^{\prime}$ be a neighbor of $w$ in $G^{\prime}$. The vertex $w^{\prime}$ is adjacent to at least one of $v_{1}$ and $v_{2}$ in $G$. Without loss of generality, we may assume that $v_{1} w^{\prime} \in E(G)$.

Suppose that $d_{G^{\prime}}(w)=1$ and $w \notin X$. Then, $\left\{v_{1}, v_{2}\right\} \cap X=\emptyset$, and so $d_{G}\left(v_{i}\right) \geq 3$ for $i=1,2$. Further, $N_{G}\left(v_{1}\right)=\left\{u_{1}, v_{2}, w^{\prime}\right\}$ and $N_{G}\left(v_{2}\right)=\left\{u_{2}, v_{1}, w^{\prime}\right\}$. If $w^{\prime} \notin X$, then the vertex $w^{\prime}$ is a $X$-cut-vertex of $G$, contradicting Claim D. Hence, $w^{\prime} \in X$. By Claim F, $w^{\prime}$ is not a cut-vertex of $G$, and so $|V(G)|=5$ and $|X|=1$. Then $\left\{u_{1}, w^{\prime}\right\}$ is an $X$-DS in $G$, and so $\gamma(G ; X)=2<20 / 8=3|V(G)| / 8+5|X| / 8=\psi(G ; X)$, as desired. Hence we may assume that $d_{G^{\prime}}(w) \geq 2$ or $w \in X$. Since $G$ is connected, so too is $G^{\prime}$. Therefore we can apply the inductive hypothesis to $G^{\prime}$.

Suppose there is a $X^{\prime}$-DS $D^{\prime}$ in $G^{\prime}$ such that $\left|D^{\prime}\right| \leq \psi\left(G^{\prime} ; X^{\prime}\right)$. If $w \in D^{\prime}$, let $D=$ $\left.\left(D^{\prime} \backslash\{w\}\right) \cup\left\{v_{1}, v_{2}\right\}\right)$. If $w \notin D^{\prime}$, then in order to dominate $w$ there must be a neighbor of $w$ in $D^{\prime}$. We may assume that $w^{\prime} \in D^{\prime}$. We now let $D=D^{\prime} \cup\left\{u_{2}\right\}$. In both cases, $|D|=\left|D^{\prime}\right|+1$ and $D$ is a $X$-DS in $G$. Thus, $\gamma(G ; X) \leq|D|=\left|D^{\prime}\right|+1 \leq \psi\left(G^{\prime} ; X^{\prime}\right)+1$. As 
$\left|V\left(G^{\prime}\right)\right|=|V(G)|-3,\left|X^{\prime}\right| \leq|X|, \operatorname{sc}\left(G^{\prime} ; X^{\prime}\right)+\mathrm{bc}\left(G^{\prime} ; X^{\prime}\right) \leq 1=\operatorname{sc}(G ; X)+\mathrm{bc}(G ; X)+1$, and $\delta_{1}\left(G^{\prime} ; X^{\prime}\right)=\delta_{1}(G ; X)=0$, we have that $\gamma(G ; X) \leq \psi\left(G^{\prime} ; X^{\prime}\right)+1 \leq[\psi(G ; X)-9 / 8+$ $1 / 8]+1=\psi(G ; X)$, as desired. Hence we may assume that there is no $X^{\prime}$-DS $D^{\prime}$ in $G^{\prime}$ such that $\left|D^{\prime}\right| \leq \psi\left(G^{\prime} ; X^{\prime}\right)$.

By induction, $X^{\prime}=X=\emptyset$ and $G^{\prime} \in \mathcal{F}$. Let $\left|V\left(G^{\prime}\right)\right|=n^{\prime}$. Then, $n^{\prime} \in\{4,7,10,13\}$. By Lemma $4(\mathrm{~b}), \gamma\left(G^{\prime}\right)=\left(n^{\prime}+2\right) / 3=(|V(G)|-1) / 3$. Let $D^{\prime}$ be a dominating set of $G^{\prime}$. If $w \in D^{\prime}$, let $\left.D=\left(D^{\prime} \backslash\{w\}\right) \cup\left\{v_{1}, v_{2}\right\}\right)$. If $w \notin D^{\prime}$, then we may assume that $w^{\prime} \in D^{\prime}$. We now let $D=D^{\prime} \cup\left\{u_{2}\right\}$. In both cases, $|D|=\left|D^{\prime}\right|+1$ and $D$ is a dominating set of $G$. Thus, $\gamma(G) \leq|D|=\left|D^{\prime}\right|+1=\gamma\left(G^{\prime}\right)+1=(|V(G)|+2) / 3$. If $n^{\prime}=13$, then $|V(G)|=16$ and $\gamma(G ; X)=\gamma(G) \leq 6=3|V(G)| / 8=\psi(G ; X)$. If $n^{\prime} \in\{4,7,10\}$, then $G \in \mathcal{F}$.

By Claim I, we may assume that $S$ is an independent set in $G$; that is, $G[S]$ consists of isolated vertices. In particular, we note that a neighbor of a vertex of $S$ in $G$ is a large vertex or belongs to $X$.

Claim J $N(S) \cap X=\emptyset$.

Proof. Let $v \in S$ and let $N(v)=\left\{w_{1}, w_{2}\right\}$. Suppose that $\left\{w_{1}, w_{2}\right\} \cap X \neq \emptyset$. We may assume that $w_{1} \in X$. Note that $w_{2} \in X$ or $d_{G}\left(w_{2}\right) \geq 3$.

Suppose that $d_{G}\left(w_{1}\right)=1$. Let $G_{2}=G-\left\{v, w_{1}\right\}$ and let $X_{2}=X \backslash\left\{w_{1}\right\}$. Then, $G_{2}$ is a connected graph such that $d_{G_{2}}(x) \geq 2$ for all $x \in V\left(G_{2}\right) \backslash X_{2}$.

Suppose there is a $X_{2}$-DS $D_{2}$ in $G_{2}$ such that $\left|D_{2}\right| \leq \psi\left(G_{2} ; X_{2}\right)$. Since $D_{2} \cup\left\{w_{1}\right\}$ is a $X$-DS in $G$, we have that $\gamma(G ; X) \leq\left|D_{2}\right|+1 \leq \psi\left(G_{2} ; X_{2}\right)+1$. As $\left|V\left(G_{2}\right)\right|=|V(G)|-2$, $\left|X_{2}\right|=|X|-1, \operatorname{sc}\left(G_{2} ; X_{2}\right)+\mathrm{bc}\left(G_{2} ; X_{2}\right) \leq 1=\operatorname{sc}(G ; X)+\mathrm{bc}(G ; X)+1$, and $\delta_{1}\left(G_{2} ; X_{2}\right)=$ $\delta_{1}(G ; X)=0$, we have that $\gamma(G ; X) \leq \psi\left(G_{2} ; X_{2}\right)+1 \leq[\psi(G ; X)-6 / 8-5 / 8+1 / 8]+1<$ $\psi(G ; X)$, as desired. Hence we may assume that $\gamma\left(G_{2} ; X_{2}\right)>\psi\left(G_{2} ; X_{2}\right)$.

By the inductive hypothesis, we must have that $X_{2}=\emptyset$ and $G_{2} \in \mathcal{F}$. By our earlier assumptions (see Claim D or Claim I), $G_{2} \neq C_{4}$. Hence, $\left|V\left(G_{2}\right)\right| \geq 7$, and so $|V(G)| \geq 9$. By Lemma 4(b), $\gamma\left(G_{2}\right) \leq\left(\left|V\left(G_{2}\right)\right|+2\right) / 3=|V(G)| / 3$. Any $\gamma\left(G_{2}\right)$-set can be extended to an $X$-DS of $G$ by adding to it the vertex $\left\{w_{1}\right\}$, and so $\gamma(G ; X) \leq \gamma\left(G_{2}\right)+1=|V(G)| / 3+1$. However since $|X|=1, \psi(G ; X)=3|V(G)| / 8+5 / 8$. Thus since $|V(G)| \geq 9$, we have that $\gamma(G ; X) \leq \psi(G ; X)$, as desired. Hence we may assume that $d_{G}\left(w_{1}\right) \geq 2$.

Let $G^{\prime}=G-v$ and let $X^{\prime}=X$. By Claim F, $w_{1}$ is not a cut-vertex of $G$. Hence, $G^{\prime}$ is connected. By the inductive hypothesis, there is a $X^{\prime}$-DS $D^{\prime}$ in $G^{\prime}$ such that $\left|D^{\prime}\right| \leq$ $\psi\left(G^{\prime} ; X^{\prime}\right)$. Since $D^{\prime}$ is a $X$-DS in $G$, we have that $\gamma(G ; X) \leq\left|D^{\prime}\right| \leq \psi\left(G^{\prime} ; X^{\prime}\right)$. As $\left|V\left(G^{\prime}\right)\right|=|V(G)|-1,\left|X^{\prime}\right|=|X|, \operatorname{sc}\left(G^{\prime} ; X^{\prime}\right)+\mathrm{bc}\left(G^{\prime} ; X^{\prime}\right) \leq 1=\operatorname{sc}(G ; X)+\mathrm{bc}(G ; X)+1$, and $\delta_{1}\left(G^{\prime} ; X^{\prime}\right)=\delta_{1}(G ; X)=0$, we have that $\gamma(G ; X) \leq\left|D^{\prime}\right| \leq \psi\left(G^{\prime} ; X^{\prime}\right) \leq \psi(G ; X)-$ $3 / 8+1 / 8<\psi(G ; X)$, as desired.

By Claim J, both neighbors of a vertex of $S$ in $G$ are large vertices and do not belong to $X$; that is, if $v \in S$ and $u \in N(v)$, then $u \notin X$ and $d_{G}(u) \geq 3$.

Claim K No two vertices of $S$ belong to a common 4-cycle that contains a vertex of degree at least 4 in $G$. 
Proof. Suppose that $v_{1} u v_{2} v v_{1}$ is a 4 -cycle in $G$ where $\{u, v\} \subseteq S$ and $d_{G}\left(v_{1}\right) \geq 4$. By Claim J, $X \cap\left\{v_{1}, v_{2}\right\}=\emptyset$. Thus by our earlier assumptions, $d_{G}\left(v_{2}\right) \geq 3$. Let $G^{\prime}=G-\{u, v\}$.

Suppose that $G^{\prime}$ is connected. Let $X^{\prime}=X \cup\left\{v_{2}\right\}$. By the inductive hypothesis, there is a $X^{\prime}$-DS $D^{\prime}$ in $G^{\prime}$ such that $\left|D^{\prime}\right| \leq \psi\left(G^{\prime} ; X^{\prime}\right)$. Since $D^{\prime}$ is a $X$-DS in $G$, we have that $\gamma(G ; X) \leq\left|D^{\prime}\right| \leq \psi\left(G^{\prime} ; X^{\prime}\right)$. As $\left|V\left(G^{\prime}\right)\right|=|V(G)|-2,\left|X^{\prime}\right|=|X|+1$, $\operatorname{sc}\left(G^{\prime} ; X^{\prime}\right)+\operatorname{bc}\left(G^{\prime} ; X^{\prime}\right) \leq 1=\operatorname{sc}(G ; X)+\operatorname{bc}(G ; X)+1$, and $\delta_{1}\left(G^{\prime} ; X^{\prime}\right)=\delta_{1}(G ; X)=0$, we have that $\gamma(G ; X) \leq \psi\left(G^{\prime} ; X^{\prime}\right) \leq \psi(G ; X)-6 / 8+5 / 8+1 / 8=\psi(G ; X)$, as desired. Hence we may assume that $G^{\prime}$ is disconnected. Let $G_{1}$ and $G_{2}$ be the two components of $G^{\prime}$, where $v_{i} \in V\left(G_{i}\right)$ for $i \in\{1,2\}$. Let $X_{1}=X \cap V\left(G_{1}\right)$.

Suppose that there is a $X_{1}$-DS $D_{1}$ in $G_{1}$ such that $\left|D_{1}\right| \leq \psi\left(G_{1} ; X_{1}\right)$. In this case, let $X_{2}=\left(X \cap V\left(G_{2}\right)\right) \cup\left\{v_{2}\right\}$. Note that for $i \in\{1,2\}, d_{G_{i}}(x) \geq 2$ for all $x \in V\left(G_{i}\right) \backslash X_{i}$. By the inductive hypothesis, there is a $X_{2}$-DS $D_{2}$ in $G_{2}$ such that $\left|D_{2}\right| \leq \psi\left(G_{2} ; X_{2}\right)$. Since $v_{2} \in$ $D_{2}$, the set $D_{1} \cup D_{2}$ is a $X$-DS in $G$, and so $\gamma(G ; X) \leq\left|D_{1}\right|+\left|D_{2}\right| \leq \psi\left(G_{1} ; X_{1}\right)+\psi\left(G_{2} ; X_{2}\right)$. As $|V(G)|=\left|V\left(G_{1}\right)\right|+\left|V\left(G_{2}\right)\right|-2,|X|=\left|X_{1}\right|+\left|X_{2}\right|+1,\left(\operatorname{sc}\left(G_{1} ; X_{1}\right)+\operatorname{bc}\left(G_{1} ; X_{1}\right)\right)+$ $\left(\operatorname{sc}\left(G_{2} ; X_{2}\right)+\mathrm{bc}\left(G_{2} ; X_{2}\right)\right) \leq \operatorname{sc}(G ; X)+\mathrm{bc}(G ; X)+1$, and $\delta_{1}\left(G_{1} ; X_{1}\right)=\delta_{1}\left(G_{2} ; X_{2}\right)=$ $\delta_{1}(G ; X)=0$, we have that $\gamma(G ; X) \leq \psi\left(G_{1} ; X_{1}\right)+\psi\left(G_{2} ; X_{2}\right) \leq \psi(G ; X)-6 / 8+5 / 8+$ $1 / 8 \leq \psi(G ; X)$, as desired. Hence we may assume that $\gamma\left(G_{1} ; X_{1}\right)>\psi\left(G_{1} ; X_{1}\right)$.

By the inductive hypothesis, we must have that $X_{1}=\emptyset$ and $G_{1} \in \mathcal{F}$. By Claim I, $G_{1} \neq C_{4}$. Hence, $\left|V\left(G_{1}\right)\right| \geq 7$. By Lemma $4(\mathrm{~b}), \gamma\left(G_{1}\right)=\left(\left|V\left(G_{1}\right)\right|+2\right) / 3$. By Lemma $5(\mathrm{~b})$, there is a $\gamma\left(G_{1}\right)$-set $S_{1}$ containing $v_{1}$. If $G_{2} \in \mathcal{F}$ and $X=\emptyset$, then $\left|V\left(G_{2}\right)\right| \geq 7$ (and so, $|V(G)| \geq 16)$ and $\gamma\left(G_{2}\right)=\left(\left|V\left(G_{2}\right)\right|+2\right) / 3$. In this case, let $S_{2}$ be a $\gamma\left(G_{2}\right)$-set and let $S=S_{1} \cup S_{2}$. Since $v_{1} \in S_{1}$, the set $S$ is a dominating set of $G$. Thus since $|V(G)|=\left|V\left(G_{1}\right)\right|+\left|V\left(G_{2}\right)\right|+2 \geq 16$, we have that $\gamma(G ; X)=\gamma(G) \leq \gamma\left(G_{1}\right)+\gamma\left(G_{2}\right)=$ $\left(\left|V\left(G_{1}\right)\right|+2\right) / 3+\left(\left|V\left(G_{2}\right)\right|+2\right) / 3=(|V(G)|+2) / 3 \leq 3|V(G)| / 8 \leq \psi(G ; X)$, as desired. Hence, we may assume that $G_{2} \notin \mathcal{F}$ or $G_{2} \in \mathcal{F}$ and $X \neq \emptyset$.

By induction, there is a $X$-DS $T_{2}$ in $G_{2}$ such that $\left|T_{2}\right| \leq \psi\left(G_{2} ; X\right)$. Since $v_{1} \in S_{1}$, the set $S_{1} \cup T_{2}$ is a $X$-DS of $G$, and so $\gamma(G ; X) \leq\left|S_{1}\right|+\left|T_{2}\right| \leq\left(\left|V\left(G_{1}\right)\right|+2\right) / 3+\psi\left(G_{2} ; X\right)$. As $\left|V\left(G_{2}\right)\right|=|V(G)|-\left|V\left(G_{1}\right)\right|-2$ and $\operatorname{sc}\left(G_{2} ; X_{2}\right)+\operatorname{bc}\left(G_{2} ; X_{2}\right)+\delta_{1}\left(G_{2} ; X_{2}\right) \leq 1=$ $\operatorname{sc}(G ; X)+\operatorname{bc}(G ; X)+\delta_{1}(G ; X)+1$, we have that $\psi\left(G_{2} ; X\right) \leq \psi(G ; X)-3\left(\left|V\left(G_{1}\right)\right|+\right.$ $2) / 8+1 / 4=\psi(G ; X)-3\left|V\left(G_{1}\right)\right| / 8-1 / 2$. Hence, $\gamma(G ; X) \leq\left(\left|V\left(G_{1}\right)\right|+2\right) / 3+\psi(G ; X)-$ $3\left|V\left(G_{1}\right)\right| / 8-1 / 2 \leq \psi(G ; X)+\left(4-\left|V\left(G_{1}\right)\right|\right) / 24<\psi(G ; X)$, as desired.

Claim L No two vertices of $S$ belong to a common 4-cycle.

Proof. Suppose that $v_{1} u v_{2} v v_{1}$ is a 4 -cycle in $G$ where $\{u, v\} \subseteq S$. By Claim J, $X \cap$ $\left\{v_{1}, v_{2}\right\}=\emptyset$. Thus by our earlier assumptions, both $v_{1}$ and $v_{2}$ are large vertices of $G$. By Claim K, $d_{G}\left(v_{1}\right)=d_{G}\left(v_{2}\right)=3$. If $v_{1} v_{2} \in E(G)$, then $G=K_{4}-e$ and the desired result follows readily. Hence we may assume that $v_{1} v_{2} \notin E(G)$. Let $N_{G}\left(v_{1}\right)=\left\{u, v, w_{1}\right\}$ and let $N_{G}\left(v_{2}\right)=\left\{u, v, w_{2}\right\}$.

Suppose $w_{1}=w_{2}$. Then $w_{1} \in X$, for otherwise $w_{1}$ would be an $X$-cut-vertex, contradicting our assumption that $\mathrm{bc}(G ; X)=0$. If $d_{G}\left(w_{1}\right) \geq 3$, then $w_{1}$ would be a cut-vertex of $G$, contradicting Claim F. Hence $d_{G}\left(w_{1}\right)=2$, and so $G=K_{2,3}$ and $|X|=1$. Thus 
$\left\{v_{1}, w_{1}\right\}$ is an $X$-DS in $G$, and so $\gamma(G ; X)=2<20 / 8=3|V(G)| / 8+5|X| / 8=\psi(G ; X)$, as desired. Hence we may assume that $w_{1} \neq w_{2}$.

Let $G^{\prime}=\left(G-\left\{u, v, v_{2}\right\}\right) \cup\left\{v_{1} w_{2}\right\}$; that is, $G$ is obtained from $G-\left\{u, v, v_{2}\right\}$ by adding the edge $v_{1} w_{2}$. Let $X^{\prime}=X$ and note that $d_{G^{\prime}}(x) \geq 2$ for all $x \in V\left(G^{\prime}\right) \backslash X^{\prime}$.

Suppose there is a $X^{\prime}$-DS $D^{\prime}$ in $G^{\prime}$ such that $\left|D^{\prime}\right| \leq \psi\left(G^{\prime} ; X^{\prime}\right)$. If $v_{1} \in D^{\prime}$, let $D=D^{\prime} \cup\left\{v_{2}\right\}$. If $v_{1} \notin D^{\prime}$ and $w_{2} \in D^{\prime}$, let $D=D^{\prime} \cup\left\{v_{1}\right\}$. If $v_{1} \notin D^{\prime}$ and $w_{2} \notin D^{\prime}$, let $D=D^{\prime} \cup\left\{v_{2}\right\}$ (note that in this case, $w_{1} \in D^{\prime}$ ). In all three cases, $D$ is an $X$-DS of $G$. Thus, $\gamma(G ; X) \leq|D|=\left|D^{\prime}\right|+1 \leq \psi\left(G^{\prime} ; X^{\prime}\right)+1$. As $\left|V\left(G^{\prime}\right)\right|=|V(G)|-3,\left|X^{\prime}\right|=|X|$, $\operatorname{sc}\left(G^{\prime} ; X^{\prime}\right)+\mathrm{bc}\left(G^{\prime} ; X^{\prime}\right) \leq 1=\operatorname{sc}(G ; X)+\mathrm{bc}(G ; X)+1$, and $\delta_{1}\left(G^{\prime} ; X^{\prime}\right)=\delta_{1}(G ; X)=0$, we have that $\gamma(G ; X) \leq \psi\left(G^{\prime} ; X^{\prime}\right)+1 \leq[\psi(G ; X)-9 / 8+1 / 8]+1=\psi(G ; X)$, as desired. Hence we may assume that $\gamma\left(G^{\prime} ; X^{\prime}\right)>\psi\left(G^{\prime} ; X^{\prime}\right)$.

By the inductive hypothesis, $G^{\prime} \in \mathcal{F}$ and $X^{\prime}=X=\emptyset$. By Lemma $5(\mathrm{c})$, there is a $\gamma\left(G^{\prime}\right)$-set $D^{\prime}$ containing both $v_{1}$ and $w_{2}$. By Lemma $4(\mathrm{~b}),\left|D^{\prime}\right|=\left(\left|V\left(G_{1}\right)\right|+2\right) / 3=$ $(|V(G)|-1) / 3$. Since the set $D^{\prime}$ is also a dominating set of $G$, we have that $\gamma(G ; X)=$ $\gamma(G) \leq\left|D^{\prime}\right|=(|V(G)|-1) / 3<3|V(G)| / 8=\psi(G ; X)$, as desired.

Recall that a set $D$ is packing in a graph if every two distinct vertices in $D$ are at distance at least 3 apart in the graph.

Claim $\mathbf{M}$ The set $S$ is a packing in $G$.

Proof. Suppose that $\{u, v\} \subseteq S$ and $d(u, v) \leq 2$. By our earlier assumptions, $S$ is an independent set, and so $d(u, v)=2$. Let $w$ be a common neighbor of $u$ and $v$. Let $N(u)=\left\{u^{\prime}, w\right\}$ and let $N(v)=\left\{v^{\prime}, w\right\}$. By Claim L, $u^{\prime} \neq v^{\prime}$. By our earlier assumptions, if $z \in N(u) \cup N(v)$, then $z \notin X$ and $d_{G}(z) \geq 3$.

Let $G^{\prime}=G-\{u, v\}$. Let $G_{u}$ and $G_{v}$ be the components of $G^{\prime}$ containing $u^{\prime}$ and $v^{\prime}$, respectively, and let $G_{w}$ be the component of $G^{\prime}$ containing $w$. We remark that the components $G_{u}, G_{v}$, and $G_{w}$ are not necessarily distinct. (One possibility, for example, is that $G^{\prime}=G_{u}=G_{v}=G_{w}$.) We proceed further with the following subclaim.

Subclaim M1 If $x \in\{u, v\}$ and $G_{x} \neq G_{w}$, then $\gamma\left(G_{x} ; X_{x}\right) \leq \psi\left(G_{x} ; X_{x}\right)$ where $X_{x}=$ $X \cap V\left(G_{x}\right)$.

Proof. For $z \in\{u, v, w\}$, let $X_{z}=X \cap V\left(G_{z}\right)$. Suppose that $G_{u} \neq G_{w}$ and that $\gamma\left(G_{u} ; X_{u}\right)>\psi\left(G_{u} ; X_{u}\right)$. Then by the inductive hypothesis, $G_{u} \in \mathcal{F}$ and $X_{u}=\emptyset$. By our earlier assumptions (see Claims I and L), $G_{u} \neq C_{4}$. Hence, $\left|V\left(G_{u}\right)\right| \geq 7$. We now consider two possibilities depending on whether $G_{u}=G_{v}$ or $G_{u} \neq G_{v}$.

Suppose $G_{u}=G_{v}$. Then, $G^{\prime}$ consists of two components, namely the component $G_{u}$, which contains both $u^{\prime}$ and $v^{\prime}$, and a component $G_{w}$ containing $w$. Thus, $|V(G)|=$ $\left|V\left(G_{u}\right)\right|+\left|V\left(G_{w}\right)\right|+2$. By Lemma $5(\mathrm{c})$, there is a $\gamma\left(G_{u}\right)$-set $D_{u}$ containing both $u^{\prime}$ and $v^{\prime}$. By Lemma 4(b), $\left|D_{u}\right|=\left(\left|V\left(G_{u}\right)\right|+2\right) / 3$. If $\gamma\left(G_{w} ; X_{w}\right)>\psi\left(G_{w} ; X_{w}\right)$, then by the inductive hypothesis, $G_{w} \in \mathcal{F}$ and $X_{w}=\emptyset$. In particular, we note that $X=\emptyset$. By our earlier assumptions, $G_{w} \neq C_{4}$. Hence, $\left|V\left(G_{w}\right)\right| \geq 7$, and so $|V(G)|=\left|V\left(G_{u}\right)\right|+\left|V\left(G_{w}\right)\right|+2 \geq 16$. Let $D_{w}$ be a $\gamma\left(G_{w}\right)$-set. By Lemma $4(\mathrm{~b}),\left|D_{w}\right|=\left(\left|V\left(G_{w}\right)\right|+2\right) / 3$. Then, $D_{u} \cup D_{w}$ is 
a dominating set of $G$, and so $\gamma(G ; X)=\gamma(G) \leq\left|D_{u}\right|+\left|D_{w}\right|=(|V(G)|+2) / 3 \leq$ $3|V(G)| / 8 \leq \psi(G ; X)$, as desired. Hence we may assume that $\gamma\left(G_{w} ; X_{w}\right) \leq \psi\left(G_{w} ; X_{w}\right)$. Now, since $X_{w}=X$ and $\operatorname{bc}\left(G_{w} ; X_{w}\right)+\operatorname{sc}\left(G_{w} ; X_{w}\right)+\delta_{1}\left(G_{w} ; X_{w}\right) \leq 1=\operatorname{bc}(G ; X)+$ $\operatorname{sc}(G ; X)+\delta_{1}(G ; X)+1$, we have that $\psi\left(G_{w} ; X_{w}\right) \leq \psi(G ; X)-3\left(\left|V\left(G_{u}\right)\right|+2\right) / 8+1 / 4=$ $\psi(G ; X)-3\left|V\left(G_{u}\right)\right| / 8-1 / 2$. Let $D_{w}$ be a $\gamma\left(G_{w} ; X_{w}\right)$-set and let $D=D_{u} \cup D_{w}$. Then, $D$ is an $X$-DS of $G$, and so $\gamma(G ; X) \leq\left|D_{u}\right|+\left|D_{w}\right| \leq \psi(G ; X)+\left(\left|V\left(G_{u}\right)\right|+2\right) / 3-$ $3\left|V\left(G_{u}\right)\right| / 8-1 / 2=\psi(G ; X)+\left(4-\left|V\left(G_{u}\right)\right|\right) / 24<\psi(G ; X)$. Hence if $G_{u}=G_{v}$, then $\gamma(G ; X) \leq \psi(G ; X)$, as desired.

Suppose that $G_{u} \neq G_{v}$. Then the edge $u u^{\prime}$ is a bridge of $G$ and $G_{u}$ is the component of $G-u u^{\prime}$ containing $u^{\prime}$. Let $D_{u}$ be a $\gamma\left(G_{u}-u^{\prime}\right)$-set. By Lemma 4(b) and Lemma 5(a), $\left|D_{u}\right|=\gamma\left(G_{u}\right)-1=\left(\left|V\left(G_{u}\right)\right|-1\right) / 3$. Let $G_{1}$ be the component of $G-u u^{\prime}$ containing $u$, and let $X_{1}=X \cup\{u\}$. By the inductive hypothesis, there is a $X_{1}$-DS $D_{1}$ in $G_{1}$ such that $\left|D_{1}\right| \leq \psi\left(G_{1} ; X_{1}\right)$. Note that $u \in D_{1}$, and so $D_{1} \cup D_{u}$ is a $X$-DS of $G$. Hence, $\gamma(G ; X) \leq\left|D_{1}\right|+\left|D_{u}\right| \leq \psi\left(G_{1} ; X_{1}\right)+\left(\left|V\left(G_{u}\right)\right|-1\right) / 3$. As $\left|V\left(G_{1}\right)\right|=|V(G)|-\left|V\left(G_{u}\right)\right|$, $\left|X_{1}\right|=|X|+1, \operatorname{sc}\left(G_{1} ; X_{1}\right)+\mathrm{bc}\left(G_{1} ; X_{1}\right)+\delta_{1}\left(G_{1} ; X_{1}\right)=\operatorname{sc}(G ; X)+\operatorname{bc}(G ; X)+\delta_{1}(G ; X)=0$, we have that $\psi\left(G_{1} ; X_{1}\right) \leq \psi(G ; X)-3\left|V\left(G_{u}\right)\right| / 8+5 / 8$. Hence, $\gamma(G ; X) \leq \psi\left(G_{1} ; X_{1}\right)+$ $\left(\left|V\left(G_{u}\right)\right|-1\right) / 3 \leq \psi(G ; X)+\left(7-\left|V\left(G_{u}\right)\right|\right) / 24 \leq \psi(G ; X)$. Hence if $G_{u} \neq G_{v}$, then $\gamma(G ; X) \leq \psi(G ; X)$, as desired.

We have shown, therefore, that if $G_{u} \neq G_{w}$ and $\gamma\left(G_{u} ; X_{u}\right)>\psi\left(G_{u} ; X_{u}\right)$, then $\gamma(G ; X) \leq \psi(G ; X)$, as desired. Hence we may assume that if $G_{u} \neq G_{w}$, then $\gamma\left(G_{u} ; X_{u}\right) \leq$ $\psi\left(G_{u} ; X_{u}\right)$

We now return to the proof of Claim M. Consider the graph $G^{\prime}=G-\{u, v\}$ defined earlier, and let $X^{\prime}=X \cup\{w\}$. Note that $d_{G^{\prime}}(x) \geq 2$ for all $x \in V\left(G^{\prime}\right) \backslash X^{\prime}$. For $z \in\{u, v, w\}$, let $X_{z}=X^{\prime} \cap V\left(G_{z}\right)$. Since $w \in X_{w}$, we have by the inductive hypothesis that $\gamma\left(G_{w} ; X_{w}\right) \leq \psi\left(G_{w} ; X_{w}\right)$. Hence by Subclaim M1, $\gamma\left(G_{u} ; X_{u}\right) \leq \psi\left(G_{u} ; X_{u}\right)$ and $\gamma\left(G_{v} ; X_{v}\right) \leq \psi\left(G_{v} ; X_{v}\right)$. It follows that $\gamma\left(G^{\prime} ; X^{\prime}\right) \leq \psi\left(G^{\prime} ; X^{\prime}\right)$. Note that $\left|V\left(G^{\prime}\right)\right|=$ $|V(G)|-2,\left|X^{\prime}\right|=|X|+1$, and $\delta_{1}\left(G^{\prime} ; X^{\prime}\right)=0$.

Subclaim M2 bc $\left(G^{\prime} ; X^{\prime}\right)+\operatorname{sc}\left(G^{\prime} ; X^{\prime}\right)=2$.

Proof. Suppose that $\mathrm{bc}\left(G^{\prime} ; X^{\prime}\right) \geq 1$. Let $x$ be a $X^{\prime}$-cut-vertex in $G^{\prime}$. Then, $G^{\prime}-x$ contains a component, $C_{x}$, which is an induced 4-cycle and which does not contain any vertices from $X^{\prime}$. Since $\mathrm{bc}(G ; X)=0$, the vertex $x$ is not a $X$-cut-vertex in $G$. Thus, $C_{x}$ must contain $u^{\prime}$ or $v^{\prime}$.

Suppose that $\operatorname{sc}\left(G^{\prime} ; X^{\prime}\right) \geq 1$. Let $C^{\prime}$ be an $X^{\prime}$-special-cycle. In particular, we note that no vertex of $C^{\prime}$ belongs to $X^{\prime}$ and that there are two consecutive vertices on $C^{\prime}$ that both have degree 2 in $G^{\prime}$. However since the set $S$ is an independent set in $G$, at least one of these two consecutive vertices on $C^{\prime}$ has degree 3 or more in $G$ and therefore must be one of $u^{\prime}$ or $v^{\prime}$.

Hence we have shown that if $G^{\prime}$ has an $X^{\prime}$-cut-vertex $x$, then the resulting induced 4-cycle in $G^{\prime}-x$ which does not contain any vertices from $X^{\prime}$ must contain $u^{\prime}$ or $v^{\prime}$, and if $G^{\prime}$ has an $X^{\prime}$-special-cycle, then it must contain $u^{\prime}$ or $v^{\prime}$. Thus, $\operatorname{bc}\left(G^{\prime} ; X^{\prime}\right)+\operatorname{sc}\left(G^{\prime} ; X^{\prime}\right) \leq 2$. If $\mathrm{bc}\left(G^{\prime} ; X^{\prime}\right)+\operatorname{sc}\left(G^{\prime} ; X^{\prime}\right) \leq 1$, then $\psi\left(G^{\prime} ; X^{\prime}\right) \leq \psi(G ; X)-6 / 8+5 / 8+1 / 8=\psi(G ; X)$. 
Since every $\gamma\left(G^{\prime} ; X^{\prime}\right)$-set is a $X$-DS of $G$, we have that $\gamma(G ; X) \leq \gamma\left(G^{\prime} ; X^{\prime}\right) \leq \psi\left(G^{\prime} ; X^{\prime}\right) \leq$ $\psi(G ; X)$, as desired. Hence we may assume that bc $\left(G^{\prime} ; X^{\prime}\right)+\operatorname{sc}\left(G^{\prime} ; X^{\prime}\right)=2$.

Subclaim M3 $\operatorname{sc}\left(G^{\prime} ; X^{\prime}\right)=0$.

Proof. Suppose that $\operatorname{sc}\left(G^{\prime} ; X^{\prime}\right) \geq 1$. Let $C^{\prime}$ be an $X^{\prime}$-special-cycle $C^{\prime}$ in $G^{\prime}$. By Subclaim M2, bc $\left(G^{\prime} ; X^{\prime}\right)+\operatorname{sc}\left(G^{\prime} ; X^{\prime}\right)=2$. As shown in the proof of Subclaim M2, the cycle $C^{\prime}$ contains exactly one of $u^{\prime}$ or $v^{\prime}$, say $u^{\prime}$. We may assume that $C^{\prime}$ is given by the cycle $u^{\prime} v_{1} v_{2} v_{3} v_{4} u^{\prime}$. Since $\operatorname{bc}(G ; X)=0$ and since $S$ is an independent set, we may assume that each of $u^{\prime}, v_{1}$ and $v_{3}$ has degree 2 in $G^{\prime}$ and that both $v_{2}$ and $v_{4}$ are large vertices in $G^{\prime}$. The degrees of the vertices in $G^{\prime}$ remain unchanged in $G$, except for $u^{\prime}, v^{\prime}$ and $w$. In particular, we note that $d_{G}\left(u^{\prime}\right)=3, d_{G}\left(v_{1}\right)=d_{G}\left(v_{3}\right)=2$, while $d_{G}\left(v_{2}\right) \geq 3$ and $d_{G}\left(v_{4}\right) \geq 3$. Thus, $\left\{v_{1}, v_{3}\right\} \subseteq S, d\left(v_{1}, v_{3}\right)=2$, and $v_{2}$ is the common neighbor of $v_{1}$ and $v_{3}$.

Let $G^{*}=G-\left\{v_{1}, v_{3}\right\}$ and let $X^{*}=X \cup\left\{v_{2}\right\}$. Proceeding as with the graph $G^{\prime}$, we may assume that $\gamma\left(G^{*} ; X^{*}\right) \leq \psi\left(G^{*} ; X^{*}\right)$. Note that $\left|V\left(G^{*}\right)\right|=|V(G)|-2,\left|X^{*}\right|=|X|+1$, and $\delta_{1}\left(G^{*} ; X^{*}\right)=0$. An identical proof as that of Subclaim M2 shows that if $G^{*}$ has an $X^{*}$-cut-vertex $x^{*}$, then the resulting induced 4-cycle in $G^{*}-x^{*}$ which does not contain any vertices from $X^{*}$ must contain $u^{\prime}$ or $v_{4}$ (the neighbors of $v_{1}$ and $v_{3}$, respectively, different from $v_{2}$ ), and if $G^{*}$ has an $X^{*}$-special-cycle, then it must contain $u^{\prime}$ or $v_{4}$. Since $u^{\prime}$ and $v_{4}$ are adjacent, it follows that $\mathrm{bc}\left(G^{*} ; X^{*}\right)+\operatorname{sc}\left(G^{*} ; X^{*}\right) \leq 1$. Thus, $\psi\left(G^{*} ; X^{*}\right) \leq$ $\psi(G ; X)-6 / 8+5 / 8+1 / 8=\psi(G ; X)$. Since every $\gamma\left(G^{*} ; X^{*}\right)$-set is a $X$-DS of $G$, we have that $\gamma(G ; X) \leq \gamma\left(G^{*} ; X^{*}\right) \leq \psi\left(G^{*} ; X^{*}\right) \leq \psi(G ; X)$, as desired. Hence we may assume that $\operatorname{sc}\left(G^{\prime} ; X^{\prime}\right)=0$.

We now return to the proof of Claim M. By Subclaims M2 and M3, bc $\left(G^{\prime} ; X^{\prime}\right)=2$ and $G^{\prime}$ contains two $X^{\prime}$-cut-vertices $x_{u}$ and $x_{v}$ such that $G^{\prime}-x_{u}$ contains an induced 4-cycle $C_{u}$ which contains the vertex $u^{\prime}$ and does not contain any vertices from $X^{\prime}$ while $G^{\prime}-x_{v}$ contains an induced 4-cycle $C_{v}$ which contains the vertex $v^{\prime}$ and does not contain any vertices from $X^{\prime}$. By definition of an $X^{\prime}$-cut-vertex, $x_{u} \notin X^{\prime}$ and $x_{v} \notin X^{\prime}$. Let $C_{u}$ be the cycle $u^{\prime} a b c u^{\prime}$ and let $C_{v}$ be the cycle $v^{\prime} d e f v^{\prime}$.

For the moment, we restrict our attention to the 4-cycle $C_{u}$ (similar arguments will hold for the 4-cycle $C_{v}$ ). The degree of the vertices $a, b$ and $c$ in $G^{\prime}$ and $G$ remain unchanged. By Claim L, $x_{u}$ is adjacent to at least two of the vertices $a, b$ and $c$ (for otherwise, $C_{u}$ would be a 4 -cycle in $G$ that contains two vertices from $S$ ).

Subclaim M4 $x_{u}$ is adjacent to exactly two of $a, b$ and $c$.

Proof. Suppose $x_{u}$ is adjacent to $a, b$ and $c$. Let $G^{*}=G-V\left(C_{u}\right)$ and let $X^{*}=$ $X \cup\left\{u, x_{u}\right\}$. Then, $d_{G^{*}}(x) \geq 2$ for all $x \in V\left(G^{*}\right) \backslash X^{*}$. Applying the inductive hypothesis to $G^{*}$ (if $G^{*}$ is connected) or to the two components of $G^{*}$ (if $G^{*}$ is disconnected), we have that $\gamma\left(G^{*} ; X^{*}\right) \leq \psi\left(G^{*} ; X^{*}\right)$. Since $\left|V\left(G^{*}\right)\right|=|V(G)|-4,\left|X^{*}\right|=|X|+2$, and $\operatorname{bc}\left(G^{*} ; X^{*}\right)+\operatorname{sc}\left(G^{*} ; X^{*}\right)+\delta_{1}\left(G^{*} ; X^{*}\right)=0$, we have that $\psi\left(G^{*} ; X^{*}\right) \leq \psi(G ; X)-12 / 8+$ $10 / 8<\psi(G ; X)$. Every $\gamma\left(G^{*} ; X^{*}\right)$-set is an $X$-DS of $G$, and so $\gamma(G ; X) \leq \gamma\left(G^{*} ; X^{*}\right)$. Therefore, $\gamma(G ; X)<\psi(G ; X)$, as desired. Hence we may assume that $x_{u}$ is adjacent to exactly two of $a, b$ and $c$. 
Subclaim M5 $x_{u}$ is not adjacent to $b$.

Proof. Suppose that $x_{u}$ is adjacent to $b$. Without loss of generality, we may assume that $x_{u}$ is adjacent to $c$. By Subclaim M4, $x_{u}$ is therefore not adjacent to $a$. Hence, $d_{G}(a)=2$ and $b c x_{u} b$ is a triangle in $G$. Let $G^{*}=G-\left\{a, u, u^{\prime}\right\}$ and $X^{*}=X$.

Suppose $\gamma\left(G^{*} ; X^{*}\right) \leq \psi\left(G^{*} ; X^{*}\right)$. Since $\left|V\left(G^{*}\right)\right|=|V(G)|-3,\left|X^{*}\right|=|X|, \operatorname{bc}\left(G^{*} ; X^{*}\right)+$ $\operatorname{sc}\left(G^{*} ; X^{*}\right) \leq 1$ and $\delta_{1}\left(G^{*} ; X^{*}\right)=0=\delta_{1}(G ; X)$, we have that $\psi\left(G^{*} ; X^{*}\right) \leq \psi(G ; X)-$ $9 / 8+1 / 8=\psi(G ; X)-1$. Every $\gamma\left(G^{*} ; X^{*}\right)$-set can be extended to a $X$-DS of $G$ by adding to it the vertex $u^{\prime}$, and so $\gamma(G ; X) \leq \gamma\left(G^{*} ; X^{*}\right)+1 \leq \psi\left(G^{*} ; X^{*}\right)+1 \leq \psi(G ; X)$, as desired. Hence we may assume that $\gamma\left(G^{*} ; X^{*}\right)>\psi\left(G^{*} ; X^{*}\right)$. Applying the inductive hypothesis to $G^{*}$, we have that at least one component of $G^{*}$ must therefore belong to the family $\mathcal{F}$ and this component of $G^{*}$ in $\mathcal{F}$ contains no vertex from $X$.

The graph $G^{*}$ has at most two components, namely a component $G_{b}$ containing the vertex $b$ and a component $G_{w}$ containing the vertex $w$. Possibly, $G_{b}=G_{w}$. Since $b c x_{u} b$ is a triangle in $G^{*}$ such that $b$ and $c$ are degree-2 vertices in $G^{*}$, by Lemma $4(\mathrm{~d}), G_{b} \notin \mathcal{F}$. Hence, $G_{b} \neq G_{w}$ and $G_{w} \in \mathcal{F}$. Further, $X \cap V\left(G_{w}\right)=\emptyset$. Note that $\left|V\left(G_{w}\right)\right| \geq 7$. By Lemma $4(\mathrm{~b})$ and Lemma $5(\mathrm{a})$, there is a set $D_{w}$ that dominates the vertices in $G_{w}-w$ such that $\left|D_{w}\right|=\gamma\left(G_{w}\right)-1=\left(\left|V\left(G_{w}\right)\right|-1\right) / 3$.

If $G_{b}=K_{3}$, then $X=\emptyset$ and $\gamma(G ; X)=\gamma(G) \leq\left|D_{w} \cup\{b, u\}\right|=\left|D_{w}\right|+2=\left(\left|V\left(G_{w}\right)\right|+\right.$ $5) / 3=(|V(G)|-1) / 3<\psi(G ; X)$, as desired. Hence we may assume that $x_{u}$ is adjacent to at least one vertex in $G_{b}$ different from $b$ and $c$. Let $G_{1}=G_{b}-c$ and let $X_{1}=$ $\left(X \cap V\left(G_{1}\right)\right) \cup\{b\}$. Then, $d_{G_{1}}(x) \geq 2$ for all $x \in V\left(G_{1}\right) \backslash X_{1}$. Note that $\left|X_{1}\right| \geq 1$. Applying the inductive hypothesis to $G_{1}$, we have that $\gamma\left(G_{1} ; X_{1}\right) \leq \psi\left(G_{1} ; X_{1}\right)$. Since $\left|V\left(G_{1}\right)\right|=$ $|V(G)|-\left|V\left(G_{w}\right)\right|-4,\left|X_{1}\right|=|X|+1$ and bc $\left(G_{1} ; X_{1}\right)+\operatorname{sc}\left(G_{1} ; X_{1}\right)+\delta_{1}\left(G_{1} ; X_{1}\right)=0$, we have that $\psi\left(G_{1} ; X_{1}\right)=\psi(G ; X)-3\left(\left|V\left(G_{w}\right)\right|+4\right) / 8+5 / 8=\psi(G ; X)-3\left|V\left(G_{w}\right)\right| / 8-7 / 8$. Every $\gamma\left(G_{1} ; X_{1}\right)$-set can be extended to a $X$-DS of $G$ by adding to it the vertices in the set $D_{w} \cup\{u\}$, and so $\gamma(G ; X) \leq \gamma\left(G_{1} ; X_{1}\right)+\left|D_{w}\right|+1 \leq\left[\psi(G ; X)-3\left|V\left(G_{w}\right)\right| / 8-7 / 8\right]+$ $\left(\left|V\left(G_{w}\right)\right|-1\right) / 3+1=\psi(G ; X)-\left(\left|V\left(G_{w}\right)\right|+5\right) / 8<\psi(G ; X)$, as desired.

We now return to the proof of Claim M. By Subclaims M5, $x_{u}$ is not adjacent to $b$ and is therefore adjacent to $a$ and $c$. In particular, we note that $d_{G}(b)=2$ and that $a b c x_{u} a$ is an induced 4-cycle in $G$ that contains no vertex of $X$. Since bc $(G ; X)=0$, we note in particular that $u^{\prime}$ is not a $X$-cut-vertex of $G$. Hence, $x_{u}$ must be adjacent to some vertex not on $C_{x}$. Possibly, $x_{u}$ is adjacent with $u^{\prime}$. Identical arguments also hold for the 4-cycle $C_{v}$. Hence we have the following subclaim:

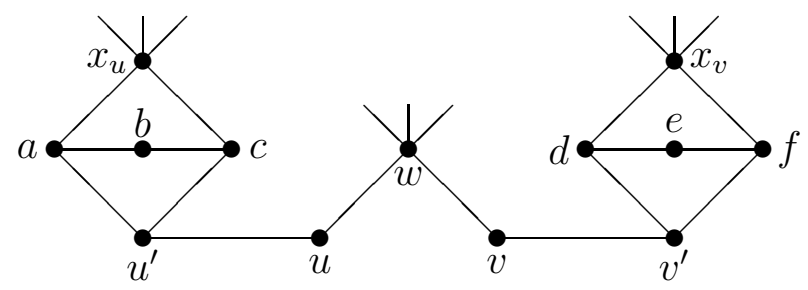

Figure 9: The graph $H_{G}$. 
Subclaim M6 The graph $H_{G}$ shown in Figure 9 is a subgraph of $G$, where the edges $u^{\prime} x_{u}$ and $v^{\prime} x_{v}$ may or may not be present in $H_{G}$ and where the degrees of the vertices of $H_{G}$ different from $x_{u}, x_{v}$ and $w$ are unchanged in $G$. Further, $V\left(H_{G}\right) \cap X=\emptyset$ and each of $x_{u}, x_{v}$ and $w$ is adjacent in $G$ to at least one vertex in $V(G) \backslash\left\{a, b, c, d, e, f, u, u^{\prime}, v, v^{\prime}\right\}$ (possibly, $w x_{u}, w x_{v}$ and $x_{u} x_{v}$ are edges of $G$ ).

We return one last time to the proof of Claim M. Let $G^{*}=(G-\{a, b, c\}) \cup\left\{u^{\prime} x_{u}\right\}$ and $X^{*}=X$. Then, $\left|V\left(G^{*}\right)\right|=|V(G)|-3$ and $G^{*}$ is a type-2 $G$-reducible graph. As shown in Lemma $2, \gamma(G ; X) \leq \gamma\left(G^{*} ; X^{*}\right)+1$.

Suppose $G^{*} \in \mathcal{F}$ and $X^{*}=\emptyset$. By Lemma 4(b), $\gamma\left(G^{*} ; X^{*}\right)=\gamma\left(G^{*}\right)=\left(\left|V\left(G^{*}\right)\right|+\right.$ $2) / 3=(|V(G)|-1) / 3$. Now either $G^{*} \in \mathcal{F}_{10} \cup \mathcal{F}_{13}$. If $G^{*} \in \mathcal{F}_{10}$, then $G \in \mathcal{F}_{13}$. Since $G$ has no bad-cut-vertex, we have that $G \in \mathcal{F}$, as desired. If $G^{*} \in \mathcal{F}_{13}$, then $|V(G)|=16$ and $\gamma(G ; X) \leq \gamma\left(G^{*} ; X^{*}\right)+1=6=3|V(G)| / 8=\psi(G ; X)$, as desired. Hence we may assume that if $G^{*} \in \mathcal{F}$, then $\left|X^{*}\right| \geq 1$. Applying the inductive hypothesis to $G^{*}$, we therefore have that $\gamma\left(G^{*} ; X^{*}\right) \leq \psi\left(G^{*} ; X^{*}\right)$. Since $\left|V\left(G^{*}\right)\right|=|V(G)|-3,\left|X^{*}\right|=|X|$, $\mathrm{bc}\left(G^{*} ; X^{*}\right)+\operatorname{sc}\left(G^{*} ; X^{*}\right) \leq 1$ and $\delta_{1}\left(G^{*} ; X^{*}\right)=0$, we have that $\psi\left(G^{*} ; X^{*}\right) \leq \psi(G ; X)-$ $9 / 8+1 / 8=\psi(G ; X)-1$. Hence, $\gamma(G ; X) \leq \gamma\left(G^{*} ; X^{*}\right)+1 \leq \psi\left(G^{*} ; X^{*}\right)+1 \leq \psi(G ; X)$, as desired. This completes the proof of Claim M.

We now return to the proof of Theorem 4. Let $u \in S$ and let $N(u)=\{v, w\}$. By Claim J, $\{v, w\} \cap X=\emptyset$. By Claim M, every vertex within distance 2 from $u$ in $G$ that does not belong to $X$ has degree at least 3 in $G$. Let $G^{\prime}=G-N[u]$ and let $X^{\prime}=X$. Let $G_{1}$ be a component of $G^{\prime}$ and let $X_{1}=X \cap V\left(G_{1}\right)$.

Claim N If $\gamma\left(G_{1} ; X_{1}\right)>\psi\left(G_{1} ; X_{1}\right)$, then $G^{\prime}=G_{1}$.

Proof. Suppose that $\gamma\left(G_{1} ; X_{1}\right)>\psi\left(G_{1} ; X_{1}\right)$ and $G^{\prime} \neq G_{1}$. Then, $G^{\prime}$ contains at least two components. Without loss of generality, we may assume that $v$ is adjacent to a vertex $v_{1}$ in $G_{1}$ and that $w$ is adjacent to a vertex in a component of $G^{\prime}$ different from $G_{1}$. By the inductive hypothesis, $G_{1} \in \mathcal{F}$ and $X_{1}=\emptyset$. Let $D_{1}$ be a $\gamma\left(G_{1}-v_{1}\right)$-set. By Lemma 4(b) and Lemma 5(a), $\left|D_{1}\right|=\gamma\left(G_{1}\right)-1=\left(\left|V\left(G_{1}\right)\right|-1\right) / 3$. Let $G^{*}=G-\left(V\left(G_{1}\right) \cup\{u\}\right)$ and let $X^{*}=X \cup\{v\}$.

Subclaim N1 $\gamma\left(G^{*} ; X^{*}\right) \leq \psi\left(G^{*} ; X^{*}\right)$.

Proof. If $G^{*}$ is connected, then applying the inductive hypothesis to $G^{*}$ we have that $\gamma\left(G^{*} ; X^{*}\right) \leq \psi\left(G^{*} ; X^{*}\right)$, as desired. Hence we may assume that $G^{*}$ is disconnected. Then, $G^{*}$ contains two components, namely a component $G_{v}$ containing the vertex $v$ and a component $G_{w}$ containing $w$. Let $X_{v}=\left(X \cap V\left(G_{v}\right)\right) \cup\{v\}$ and let $X_{w}=X \cap V\left(G_{w}\right)$.

Suppose that $\gamma\left(G_{w} ; X_{w}\right)>\psi\left(G_{w} ; X_{w}\right)$. By the inductive hypothesis, $G_{w} \in \mathcal{F}$ and $X_{w}=\emptyset$. Since $S$ is an independent set, $G_{w} \neq C_{4}$. Hence, $\left|V\left(G_{w}\right)\right| \geq 7$. Let $D_{w}$ be a $\gamma\left(G_{w}-w\right)$-set. By Lemma 4(b) and Lemma 5(a), $\left|D_{w}\right|=\gamma\left(G_{w}\right)-1=\left(\left|V\left(G_{w}\right)\right|-1\right) / 3$. We now consider the graph $G_{u}=G-V\left(G_{w}\right)$ and let $X_{u}=X \cup\{u\}$. By the inductive hypothesis, there is a $X_{u}$-DS $D_{u}$ in $G_{u}$ such that $\left|D_{u}\right| \leq \psi\left(G_{u} ; X_{u}\right)$. Note that $D_{u} \cup D_{w}$ 
is a $\gamma(G ; X)$-DS of $G$. Hence, $\gamma(G ; X) \leq\left|D_{u}\right|+\left|D_{w}\right| \leq \psi\left(G_{u} ; X_{u}\right)+\left(\left|V\left(G_{w}\right)\right|-1\right) / 3$. As $\left|V\left(G_{u}\right)\right|=|V(G)|-\left|V\left(G_{w}\right)\right|,\left|X_{u}\right|=|X|+1, \operatorname{sc}\left(G_{u} ; X_{u}\right)+\operatorname{bc}\left(G_{u} ; X_{u}\right)+\delta_{1}\left(G_{u} ; X_{u}\right)=$ $\operatorname{sc}(G ; X)+\operatorname{bc}(G ; X)+\delta_{1}(G ; X)=0$, we have that $\psi\left(G_{u} ; X_{u}\right) \leq \psi(G ; X)-3\left|V\left(G_{w}\right)\right| / 8+$ 5/8. Hence, $\gamma(G ; X) \leq \psi\left(G_{u} ; X_{u}\right)+\left(\left|V\left(G_{w}\right)\right|-1\right) / 3 \leq \psi(G ; X)+\left(7-\left|V\left(G_{w}\right)\right|\right) / 24 \leq$ $\psi(G ; X)$. Hence if $\gamma\left(G_{w} ; X_{w}\right)>\psi\left(G_{w} ; X_{w}\right)$, then $\gamma(G ; X) \leq \psi(G ; X)$, as desired.

Therefore we may assume that $\gamma\left(G_{w} ; X_{w}\right) \leq \psi\left(G_{w} ; X_{w}\right)$. Applying the inductive hypothesis to $G_{v}, \gamma\left(G_{v} ; X_{v}\right) \leq \psi\left(G_{v} ; X_{v}\right)$. Thus, $\gamma\left(G^{*} ; X^{*}\right)=\gamma\left(G_{v} ; X_{v}\right)+\gamma\left(G_{w} ; X_{w}\right) \leq$ $\psi\left(G_{v} ; X_{v}\right)+\psi\left(G_{w} ; X_{w}\right)=\psi\left(G^{*} ; X^{*}\right)$, as claimed.

Note that it is possible that the vertex $w$ belongs to an $X^{*}$-special-cycle in $G^{*}$. Further, note that the vertex $w$ has degree at least 3 in $G$ and therefore at least 2 in $G^{*}$. Hence since $\left|V\left(G^{*}\right)\right|=|V(G)|-\left|V\left(G_{1}\right)\right|-1,\left|X^{*}\right|=|X|+1, \operatorname{bc}\left(G^{*} ; X^{*}\right)+\operatorname{sc}\left(G^{*} ; X^{*}\right) \leq 1$ and $\delta_{1}\left(G^{*} ; X^{*}\right)=\delta_{1}(G ; X)=0$, we have that $\psi\left(G^{*} ; X^{*}\right) \leq \psi(G ; X)-3\left(\left|V\left(G_{1}\right)\right|+1\right) / 8+$ $5 / 8+1 / 8=\psi(G ; X)-3\left(\left|V\left(G_{1}\right)\right|-1\right) / 8$. Every $\gamma\left(G^{*} ; X^{*}\right)$-set can be extended to a $X$-DS of $G$ by adding to it the vertices in the set $D_{1}$. Hence, $\gamma(G ; X) \leq \gamma\left(G^{*} ; X^{*}\right)+\left|D_{1}\right|=$ $\psi(G ; X)-3\left(\left|V\left(G_{1}\right)\right|-1\right) / 8+\left(\left|V\left(G_{1}\right)\right|-1\right) / 3=\psi(G ; X)+\left(1-\left|V\left(G_{1}\right)\right|\right) / 24<\psi(G ; X)$, as desired. Thus we have shown that if $\gamma\left(G_{1} ; X_{1}\right)>\psi\left(G_{1} ; X_{1}\right)$ and $G^{\prime} \neq G_{1}$, then $\gamma(G ; X) \leq \psi(G ; X)$.

Claim O $\gamma\left(G^{\prime} ; X^{\prime}\right) \leq \psi\left(G^{\prime} ; X^{\prime}\right)$.

Proof. Suppose that $\gamma\left(G^{\prime} ; X^{\prime}\right)>\psi\left(G^{\prime} ; X^{\prime}\right)$. We may assume that $\gamma\left(G_{1} ; X_{1}\right)>\psi\left(G_{1} ; X_{1}\right)$. By Claim $\mathrm{N}, G^{\prime}=G_{1}$. In particular, note that $G_{1}$ is connected. By the inductive hypothesis, $G_{1} \in \mathcal{F}$ and $X_{1}=X=\emptyset$. Equivalently, $G^{\prime} \in \mathcal{F}$ and $X^{\prime}=\emptyset$. Hence, $\left|V\left(G^{\prime}\right)\right| \geq 4$ and $V(G)=V\left(G^{\prime}\right) \cup\{u, v, w\}$. Further, $\gamma(G ; X)=\gamma(G)$ and $\psi(G ; X)=$ $3|V(G)| / 8$. We wish therefore to show that $\gamma(G) \leq 3|V(G)| / 8$. If $\{v, w\}$ dominates $V(G)$, then $\gamma(G)=2<3|V(G)| / 8$, as desired. Hence we may assume that at least one vertex in $V\left(G^{\prime}\right)$ is not dominated by $\{v, w\}$ in $G$.

Subclaim O1 vw is not an edge of $G$.

Proof. Suppose $v w$ is an edge of $G$. Let $v_{1}$ be a neighbor of $v$ different from $u$ and $w$. Let $D^{\prime}$ be a $\gamma\left(G^{\prime}-v_{1}\right)$-set. By Lemma $4(\mathrm{~b})$ and Lemma $5(\mathrm{a}),\left|D^{\prime}\right|=\gamma\left(G^{\prime}\right)-1=$ $\left(\left|V\left(G^{\prime}\right)\right|-1\right) / 3=(|V(G)|-4) / 3$. Since $v w$ is an edge of $G$, the set $D^{\prime} \cup\{v\}$ is a dominating set of $G$, and so $\gamma(G) \leq\left|D^{\prime}\right|+1=(|V(G)|-1) / 3<3|V(G)| / 8$, as desired.

Subclaim O2 $N(v) \cap N(w)=\{u\}$.

Proof. Suppose there is a vertex $u^{\prime} \in V\left(G^{\prime}\right)$ that is adjacent to both $v$ and $w$ in $G$. Let $G^{\prime \prime}$ be the graph obtained from $G$ by deleting all edges from $\{v, w\}$ to $V(G) \backslash\left\{u, u^{\prime}\right\}$. Thus, $G^{\prime \prime}$ is obtained from $G^{\prime}$ by adding the vertices $\{u, v, w\}$ and the edges $\left\{u v, u w, u^{\prime} v, u^{\prime} w\right\}$. Note that $u^{\prime} v u w$ is a path in $G^{\prime \prime}$ and that each of $u, v$ and $w$ has degree 2 in $G^{\prime \prime}$. Since $G^{\prime}$ is a type- $1 G^{\prime \prime}$-reducible graph and $G^{\prime} \in \mathcal{F}$, the graph $G^{\prime \prime} \in \mathcal{F}$. By Lemma 1, $\gamma\left(G^{\prime \prime}\right)=\gamma\left(G^{\prime}\right)+1=(|V(G)|-1) / 3+1$. If we now restore the graph $G$ from $G^{\prime \prime}$ by adding the deleted edges incident with $v$ or $w$, we have that either $G \in \mathcal{F}$ or, by repeated 
applications of Lemma $5(\mathrm{~d})$, we have that $\gamma(G) \leq \gamma\left(G^{\prime \prime}\right)-1=(|V(G)|-1) / 3<3|V(G)| / 8$, as desired.

Let $v^{\prime} \in N(v) \backslash\{u\}$ and let $w^{\prime} \in N(w) \backslash\{u\}$. By Subclaim O2, $v^{\prime} \neq w^{\prime}$.

Subclaim O3 $v^{\prime} w^{\prime} \notin E\left(G^{\prime}\right)$.

Proof. Suppose $v^{\prime} w^{\prime} \in E\left(G^{\prime}\right)$. Let $G^{\prime \prime}$ be the graph obtained from $G$ by deleting all edges from $\{v, w\}$ to $V(G) \backslash\left\{u, v^{\prime}, w^{\prime}\right\}$. Thus, $G^{\prime \prime}$ is obtained from $G^{\prime}$ by adding the vertices $\{u, v, w\}$ and the edges $\left\{u v, u w, v v^{\prime}, w w^{\prime}\right\}$. Note that $v^{\prime} v u w w^{\prime} v^{\prime}$ is a cycle in $G^{\prime \prime}$ and that each of $u, v$ and $w$ has degree 2 in $G^{\prime \prime}$. Since $G^{\prime}$ is a type- $1 G^{\prime \prime}$-reducible graph and $G^{\prime} \in \mathcal{F}$, the graph $G^{\prime \prime} \in \mathcal{F}$. An identical argument as that in the proof of Subclaim O2, shows that either $G \in \mathcal{F}$ or $\gamma(G)<3|V(G)| / 8$, as desired.

Subclaim O4 $G^{\prime}+v^{\prime} w^{\prime} \in \mathcal{F}$.

Proof. Suppose $G^{\prime}+v^{\prime} w^{\prime} \notin \mathcal{F}$. Let $D^{\prime}$ be a $\gamma\left(G^{\prime}+v^{\prime} w^{\prime}\right)$-set. By Lemma 4(b) and Lemma 5(d), $\left|D^{\prime}\right|=\gamma_{t}\left(G^{\prime}\right)-1=(|V(G)|-4) / 3$. Since $\gamma\left(G^{\prime}+v^{\prime} w^{\prime}\right)<\gamma\left(G^{\prime}\right), \mid D \cap$ $\left\{v^{\prime}, w^{\prime}\right\} \mid=1$. We may assume that $v^{\prime} \in D^{\prime}$. Hence, $D^{\prime} \cup\{w\}$ is a dominating set of $G$, and so $\gamma(G) \leq\left|D^{\prime}\right|+1=(|V(G)|-1) / 3<3|V(G)| / 8$, as desired.

We now return to the proof of Claim O. Let $G^{\prime \prime}$ be the graph obtained from $G$ by deleting all edges from $\{v, w\}$ to $V(G) \backslash\left\{u, v^{\prime}, w^{\prime}\right\}$. Thus, $G^{\prime \prime}$ is obtained from $G^{\prime}$ by adding the vertices $\{u, v, w\}$ and the edges $\left\{u v, u w, v v^{\prime}, w w^{\prime}\right\}$. Note that $v^{\prime} v u w w^{\prime}$ is a path in $G^{\prime \prime}$ and that each of $u, v$ and $w$ has degree 2 in $G^{\prime \prime}$. Since $G^{\prime}+v^{\prime} w^{\prime}$ is a type-1 $G^{\prime \prime}$-reducible graph and since, by Subclaim O4, $G^{\prime}+v^{\prime} w^{\prime} \in \mathcal{F}$, the graph $G^{\prime \prime} \in \mathcal{F}$. An identical argument as that in the proof of Subclaim O2, shows that either $G \in \mathcal{F}$ or $\gamma(G)<3|V(G)| / 8$, as desired. This completes the proof of Claim O.

Recall that $G^{\prime}=G-N[u]$ and $X^{\prime}=X$. Further, $G_{1}$ denotes a component of $G^{\prime}$ and $X_{1}=X \cap V\left(G_{1}\right)$.

Claim $\mathbf{P} \operatorname{sc}\left(G^{\prime} ; X^{\prime}\right)=0$.

Proof. Suppose that $\operatorname{sc}\left(G^{\prime} ; X^{\prime}\right) \geq 1$. We may assume that $G_{1}$ contains an $X_{1}$-specialcycle $C: v_{1} v_{2} v_{3} v_{4} v_{5} v_{1}$. In particular, we note that $V(C) \cap X_{1}=\emptyset$. Renaming vertices if necessary, we may assume that $v_{2}, v_{4}, v_{5}$ are degree- 2 vertices in $G_{1}$. Possibly, $v_{1}$ or $v_{3}$ or both $v_{1}$ and $v_{3}$ are large vertices, and possibly $v_{1} v_{3}$ is an edge of $G_{1}$. Since $S$ is a packing in $G$, at most one vertex in $V(C)$ has degree 2 in $G$.

Claim P1 Every vertex in $C$ has degree at least 3 in $G$.

Proof. Suppose that the cycle $C$ contains a vertex $v^{*}$ that has degree- 2 in $G$. Without loss of generality, we may assume that $v^{*} \in\left\{v_{2}, v_{5}\right\}$. Since $S$ is a packing in $G$, every vertex in $V(C) \backslash\left\{v^{*}\right\}$ has degree at least 3 in $G$. By assumption, each vertex in $\left\{v_{2}, v_{4}, v_{5}\right\} \backslash\left\{v^{*}\right\}$ has degree 2 in $G_{1}$ and is therefore dominated by the set $\{v, w\}$. Let $G^{*}=G-\left\{v_{2}, v_{5}\right\}$ and let 
$X^{*}=X \cup\left\{v_{1}\right\}$. Then, $d_{G^{*}}(x) \geq 2$ for all $x \in V\left(G^{*}\right) \backslash X^{*}$. In particular, $\delta_{1}\left(G^{*} ; X^{*}\right)=0$. Let $W=\left\{u, v, v_{3}, v_{4}, w\right\}$. Since $v_{4}$ is dominated by $\{v, w\}, G[W]$ is connected and contains a path $P_{5}$ as a subgraph. By our earlier assumptions, $\operatorname{bc}(G ; X)+\operatorname{sc}(G ; X)=0$. Hence if $G^{*}$ has an $X^{*}$-special-cycle, then such a cycle must contain at least one vertex from the set $W$, while if $G^{*}$ has an $X^{*}$-cut-vertex $x^{*}$, then the resulting 4-cycle component in $G^{*}-x^{*}$ must contain at least one vertex from the set $W$. From this we can deduce that $\mathrm{bc}\left(G^{*} ; X^{*}\right)+\operatorname{sc}\left(G^{*} ; X^{*}\right) \leq 1$.

We show that we may assume $\gamma\left(G^{*} ; X^{*}\right) \leq \psi\left(G^{*} ; X^{*}\right)$. If $G^{*}$ is connected, then by the inductive hypothesis, $\gamma\left(G^{*} ; X^{*}\right) \leq \psi\left(G^{*} ; X^{*}\right)$ since $\left|X^{*}\right| \geq 1$. Suppose $G^{*}$ is disconnected. Then, $G^{*}$ contains exactly two components, namely a component $F$ containing $v_{1}$ and a component $H$ containing the vertices in the set $W$. Let $X_{F}=X^{*} \cap V(F)$ and let $X_{H}=X^{*} \cap V(H)$. Note that $v_{1} \in X_{F}$ and $\gamma\left(F ; X_{F}\right) \leq \psi\left(F ; X_{F}\right)$ by applying the inductive hypothesis to $F$. If $\gamma\left(H ; X_{H}\right) \leq \psi\left(H ; X_{H}\right)$, then $\gamma\left(G^{*} ; X^{*}\right)=\gamma\left(F ; X_{F}\right)+$ $\gamma\left(H ; X_{H}\right) \leq \psi\left(F ; X_{F}\right)+\psi\left(H ; X_{H}\right)=\psi\left(G^{*} ; X^{*}\right)$, as desired. Hence we may assume that $\gamma\left(H ; X_{H}\right)>\psi\left(H ; X_{H}\right)$. By the inductive hypothesis, $H \in \mathcal{F}$ and $X_{H}=\emptyset$. Note that $|V(H)| \geq 7$. By Lemma $5(\mathrm{c})$, there is a $\gamma(H)$-set $D_{H}$ that contains both $v_{3}$ and $v_{4}$. By Lemma 4 (b), $\left|D_{H}\right|=(|V(H)|+2) / 3$. We now let $I=F$ and $X_{I}=X \cap V(I)=X$. Thus, $X_{I}=X_{F} \backslash\left\{v_{1}\right\}$. Then, $\operatorname{bc}\left(I ; X_{I}\right)+\operatorname{sc}\left(I ; X_{I}\right)+\delta_{1}\left(I ; X_{I}\right) \leq 1$. If $\gamma\left(I ; X_{I}\right) \leq \psi\left(I ; X_{I}\right)$, then $\gamma(G ; X) \leq \gamma\left(I ; X_{I}\right)+\left|D_{H}\right| \leq \psi\left(I ; X_{I}\right)+\left|D_{H}\right|=[\psi(G ; X)-3(|V(H)|+2) / 8+1 / 4]+$ $(|V(H)|+2) / 3=\psi(G ; X)+(4-|V(H)|) / 24<\psi(G ; X)$, as desired. Hence we may assume that $\gamma\left(I ; X_{I}\right)>\psi\left(I ; X_{I}\right)$. By the inductive hypothesis, $I \in \mathcal{F}$ and $X_{I}=\emptyset$. By our earlier assumptions, $|V(I)| \geq 7$, and so $|V(G)| \geq 16$. Let $D_{I}$ be a $\gamma(I)$-set. By Lemma $4(\mathrm{~b})$, $\left|D_{I}\right|=(|V(I)|+2) / 3=(|V(G)|-|V(H)|) / 3$. In this case, $D_{H} \cup D_{I}$ is a dominating set of $G$, and so $\gamma(G ; X)=\gamma(G) \leq\left|D_{H}\right|+\left|D_{I}\right| \leq(|V(G)|-|V(H)|) / 3+(|V(H)|+2) / 3=$ $(|V(G)|+2) / 3 \leq 3|V(G)| / 8=\psi(G ; X)$, as desired. Hence we have shown that we may assume $\gamma\left(G^{*} ; X^{*}\right) \leq \psi\left(G^{*} ; X^{*}\right)$.

Since $\left|V\left(G^{*}\right)\right|=|V(G)|-2,\left|X^{*}\right|=|X|+1, \operatorname{bc}\left(G^{*} ; X^{*}\right)+\operatorname{sc}\left(G^{*} ; X^{*}\right) \leq 1$ and $\delta_{1}\left(G^{*} ; X^{*}\right)=0$, we have that $\psi\left(G^{*} ; X^{*}\right) \leq \psi(G ; X)-6 / 8+5 / 8+1 / 8=\psi(G ; X)$. Since every $\gamma\left(G^{*} ; X^{*}\right)$-set is an $X$-DS of $G$, we have that $\gamma(G ; X) \leq \gamma\left(G^{*} ; X^{*}\right) \leq \psi\left(G^{*} ; X^{*}\right) \leq$ $\psi(G ; X)$, as desired. Hence we may assume that every vertex in $C$ has degree at least 3 in $G$.

By Claim P1, $\{v, w\}$ dominates the set $\left\{v_{2}, v_{4}, v_{5}\right\}$. Let $Y=\left\{v_{2}, v_{4}, v_{5}\right\}$. We may assume that $|N(w) \cap Y| \geq|N(v) \cap Y|$. If $w$ dominates $Y$, then let $w^{*}$ be an arbitrary vertex in $Y$; otherwise, let $w^{*}$ be a vertex in $Y$ that is adjacent to $v$ but not to $w$. Without loss of generality, we may assume that $w^{*} \in\left\{v_{2}, v_{5}\right\}$, and so $v_{1} w^{*} \in E(G)$. Further we may assume that $v$ is adjacent to $w^{*}$ if $v$ has a neighbor in $Y$. Thus if $v$ is not adjacent to $w^{*}$, then $N(v) \cap Y=\emptyset$ (in which case $w$ dominates $Y$ ). We now let $G^{*}=G-\left\{u, w^{*}\right\}$. If $v$ is adjacent to $w^{*}$, we let $X^{*}=X \cup\{v\}$; otherwise, we let $X^{*}=X \cup\{w\}$. Then, $d_{G^{*}}(x) \geq 2$ for all $x \in V\left(G^{*}\right) \backslash X^{*}$.

Claim P2 $\gamma\left(G^{*} ; X^{*}\right) \leq \psi\left(G^{*} ; X^{*}\right)$.

Proof. If $G^{*}$ is connected, then by the inductive hypothesis, $\gamma\left(G^{*} ; X^{*}\right) \leq \psi\left(G^{*} ; X^{*}\right)$ since $\left|X^{*}\right| \geq 1$. Hence we may assume that $G^{*}$ is disconnected. Then, $G^{*}$ contains exactly two 
components, namely a component $F$ containing $v$ and a component $H$ containing $w$. Since $|N(w) \cap Y| \geq|N(v) \cap Y|$, our choice of $w^{*}$ implies that $V(C) \backslash\left\{w^{*}\right\} \subset V(H)$ and $Y \backslash\left\{w^{*}\right\} \subseteq N(w)$. Let $X_{F}=X^{*} \cap V(F)$ and let $X_{H}=X^{*} \cap V(H)$. Since $d_{G}(v) \geq 3$, we have that $d_{F}(v) \geq 1$ if $x \in X_{F}$ and $d_{F}(v) \geq 2$ if $x \notin X_{F}$.

If $v \in X_{F}$, then $\gamma\left(F ; X_{F}\right) \leq \psi\left(F ; X_{F}\right)$ by applying the inductive hypothesis to $F$. If $x \notin X_{F}$, then $w \in X_{H}$ and $\gamma\left(H ; X_{H}\right) \leq \psi\left(H ; X_{H}\right)$ by applying the inductive hypothesis to $H$. If $\gamma\left(F ; X_{F}\right) \leq \psi\left(F ; X_{F}\right)$ and $\gamma\left(H ; X_{H}\right) \leq \psi\left(H ; X_{H}\right)$, then $\gamma\left(G^{*} ; X^{*}\right) \leq \psi\left(G^{*} ; X^{*}\right)$, as desired. Hence we may assume that $\gamma\left(F ; X_{F}\right)>\psi\left(F ; X_{F}\right)$ or $\gamma\left(H ; X_{H}\right)>\psi\left(H ; X_{H}\right)$.

Suppose that $\gamma\left(F ; X_{F}\right)>\psi\left(F ; X_{F}\right)$. Then, $w \in X_{H}$ and $\gamma\left(H ; X_{H}\right) \leq \psi\left(H ; X_{H}\right)$. By the inductive hypothesis, $F \in \mathcal{F}$ and $X_{F}=\emptyset$. Note that $|V(F)| \geq 7$. By Lemma $5(\mathrm{~b})$, there is a $\gamma(F)$-set $D_{F}$ that contains $v$. By Lemma $4(\mathrm{~b}),\left|D_{F}\right|=(|V(F)|+2) / 3$. We now let $I=H$ and $X_{I}=X \cap V(I)=X$. Thus, $X_{I}=X_{H} \backslash\{w\}$. Then, $\operatorname{bc}\left(I ; X_{I}\right)+\operatorname{sc}\left(I ; X_{I}\right) \leq 1$ and $\delta_{1}\left(I ; X_{I}\right)=0$. Proceeding now as in the second paragraph of the proof of Claim P1, we can show that $\gamma\left(G^{*} ; X^{*}\right) \leq \psi\left(G^{*} ; X^{*}\right)$, as desired.

Suppose that $\gamma\left(H ; X_{H}\right)>\psi\left(H ; X_{H}\right)$. Then, $v \in X_{F}$ and $\gamma\left(F ; X_{F}\right) \leq \psi\left(F ; X_{F}\right)$. By the inductive hypothesis, $H \in \mathcal{F}$ and $X_{H}=\emptyset$. Note that $|V(H)| \geq 7$. By Lemma $5(\mathrm{c})$, there is a $\gamma(H)$-set $D_{H}$ that contains both $v_{1}$ and $w$. Note that $\left\{v_{1}, w\right\}$ dominates $\left\{u, w^{*}\right\}$ in $G$. By Lemma 4(b), $\left|D_{H}\right|=(|V(H)|+2) / 3$. We now let $I=F$ and $X_{I}=X \cap V(I)=X$. Thus, $X_{I}=X_{F} \backslash\left\{v_{1}\right\}$. Then, $\operatorname{bc}\left(I ; X_{I}\right)+\operatorname{sc}\left(I ; X_{I}\right)+\delta_{1}\left(I ; X_{I}\right) \leq 1$. Proceeding now as in the second paragraph of the proof of Claim P1, we can show that $\gamma\left(G^{*} ; X^{*}\right) \leq \psi\left(G^{*} ; X^{*}\right)$, as desired.

Claim P3 Both $v_{1}$ and $v_{3}$ are adjacent in $G_{1}$ to at least one vertex not on $C$.

Proof. Suppose $v_{1}$ or $v_{3}$, say $v_{1}$, is adjacent in $G_{1}$ only to vertices in $C$. Then, $N_{G_{1}}\left(v_{1}\right)=$ $\left\{v_{2}, v_{3}, v_{5}\right\}$. Let $K=G-\left\{u, v_{1}, v_{2}, v_{4}, v_{5}\right\}$ and let $X_{K}=X \cup\left\{v, v_{3}, w\right\}$. Then, $|V(K)|=$ $|V(G)|-5,\left|X_{K}\right|=|X|+3$, and $\mathrm{bc}\left(K ; X_{K}\right)+\operatorname{sc}\left(K ; X_{K}\right)+\delta_{1}\left(K ; X_{K}\right)=0$. Applying the inductive hypothesis to $K$ or to the components of $K$, we have that $\gamma(G ; X) \leq$ $\gamma\left(K ; X_{K}\right) \leq \psi\left(K ; X_{K}\right) \leq \psi(G ; X)-5 *(3 / 8)+3 *(5 / 8)=\psi(G ; X)$, as desired.

We now return to the proof of Claim P. Recall that $G^{*}=G-\left\{u, w^{*}\right\}$ and that $X^{*}=X \cup\{v\}$ if $v$ is adjacent to $w^{*}$ and $X^{*}=X \cup\{w\}$ if $v$ is not adjacent to $w^{*}$ (in which case, $N(v) \cap Y=\emptyset)$. Note that $\left|V\left(G^{*}\right)\right|=|V(G)|-2,\left|X^{*}\right|=|X|+1$, and $\delta_{1}\left(G^{*} ; X^{*}\right)=0$. By Claim P3 and since bc $(G ; X)+\operatorname{sc}(G ; X)=0$, we have that bc $\left(G^{*} ; X^{*}\right)+\operatorname{sc}\left(G^{*} ; X^{*}\right) \leq 1$. Hence by Claim P2, $\gamma\left(G^{*} ; X^{*}\right) \leq \psi\left(G^{*} ; X^{*}\right) \leq \psi(G ; X)-6 / 8+5 / 8+1 / 8 \leq \psi(G ; X)$. Since every $\gamma\left(G^{*} ; X^{*}\right)$-set is an $X$-DS of $G$, we have that $\gamma(G ; X) \leq \gamma\left(G^{*} ; X^{*}\right) \leq \psi\left(G^{*} ; X^{*}\right) \leq$ $\psi(G ; X)$, as desired. This completes the proof of Claim P.

Claim Q The degree-2 vertex $u$ can be chosen so that $\mathrm{bc}\left(G^{\prime} ; X^{\prime}\right)=0$.

Proof. Suppose that $\mathrm{bc}\left(G^{\prime} ; X^{\prime}\right) \geq 1$. We may assume that $\mathrm{bc}\left(G_{1} ; X_{1}\right) \geq 1$. Let $x$ be a $X_{1}$-cut-vertex in $G_{1}$. Then, $x \notin X_{1}$ and $G_{1}-x$ contains a component, $C_{x}$, which is an induced 4-cycle and which does not contain any vertices from $X_{1}$. Furthermore $x$ is adjacent to at least one but at most three vertices on $C_{x}$. Since bc $(G ; X)=0$, the vertex $x$ is not a $X$-cut-vertex in $G$. Thus, at least one vertex in $C_{x}$ is adjacent to $v$ or $w$ in $G$. 
For each vertex $z$ in $C_{x}$, let $z^{\prime}$ denote the vertex on $C_{x}$ that is not adjacent with $z$. Let $C_{x}$ be the cycle $v_{1} v_{2} v_{3} v_{4} v_{1}$. Thus, $v_{1}^{\prime}=v_{3}, v_{2}^{\prime}=v_{4}, v_{3}^{\prime}=v_{1}$ and $v_{4}^{\prime}=v_{2}$. We proceed further with the following subclaim.

Subclaim Q1 $C_{x}$ contains a degree-2 vertex in $G$.

Proof. Suppose that every vertex of $C_{x}$ has degree at least 3 in $G$. This implies that $\{v, w, x\}$ dominates the set $V\left(C_{x}\right)$ in $G$. Let $G^{*}=G-\left\{u, v_{1}, v_{2}, v_{3}, v_{4}\right\}$ and let $X^{*}=$ $X \cup\{v, w, x\}$. Every vertex in $V\left(G^{*}\right) \backslash X^{*}$ has degree at least 2 in $G^{*}$. Applying the inductive hypothesis to each component in $G^{*}$, we have that $\gamma\left(G^{*} ; X^{*}\right) \leq \psi\left(G^{*} ; X^{*}\right)$. Note that $\left|V\left(G^{*}\right)\right|=|V(G)|-5,\left|X^{*}\right|=|X|+3$ and $\operatorname{bc}\left(G^{*} ; X^{*}\right)+\operatorname{sc}\left(G^{*} ; X^{*}\right)+\delta_{1}\left(G^{*} ; X^{*}\right)=0$. Hence, $\psi\left(G^{*} ; X^{*}\right) \leq \psi(G ; X)-5 *(3 / 8)+3 *(5 / 8)=\psi(G ; X)$. Since every $\gamma\left(G^{*} ; X^{*}\right)$-set is an $X$-DS of $G$, we have that $\gamma(G ; X) \leq \gamma\left(G^{*} ; X^{*}\right) \leq \psi\left(G^{*} ; X^{*}\right) \leq \psi(G ; X)$, as desired.

Subclaim Q2 $\left|V\left(G_{1}\right)\right| \geq 6$.

Proof. Suppose that $V\left(G_{1}\right)=V\left(C_{x}\right) \cup\{x\}$. Let $G^{*}=G-\left(V\left(G_{1}\right) \cup\{u\}\right)$ and let $X^{*}=X \cup\{v, w\}$. Applying the inductive hypothesis to $G^{*}$, we have that $\gamma\left(G^{*} ; X^{*}\right) \leq$ $\psi\left(G^{*} ; X^{*}\right)$. Let $X^{*}$ be an $X^{*}$-DS of $G^{*}$. Since $\left|V\left(G^{*}\right)\right|=|V(G)|-6,\left|X^{*}\right|=|X|+2$, $\mathrm{bc}\left(G^{*} ; X^{*}\right)+\operatorname{sc}\left(G^{*} ; X^{*}\right)+\delta_{1}\left(G^{*} ; X^{*}\right)=0$, we have that $\psi\left(G^{*} ; X^{*}\right)=\psi(G ; X)-18 / 8+$ $10 / 8=\psi(G ; X)-1$. Renaming vertices, if necessary, we may assume that $x$ is adjacent with $v_{1}$. Let $v^{*} \in V\left(G_{1}\right)$. If $\left\{v, v^{*}, w\right\}$ dominates $V\left(G_{1}\right)$, then $X^{*} \cup\left\{v^{*}\right\}$ is a $X$-DS of $G$, and so $\gamma(G ; X) \leq\left|X^{*}\right|+1=\gamma\left(G^{*} ; X^{*}\right)+1 \leq \psi\left(G^{*} ; X^{*}\right)+1 \leq \psi(G ; X)$, as desired. Hence we may assume that if $v^{*} \in V\left(G_{1}\right)$, then $\left\{v, v^{*}, w\right\}$ does not dominate $V\left(G_{1}\right)$.

As observed earlier, at least one vertex in $C_{x}$ is adjacent to $v$ or $w$ in $G$. If $x$ is adjacent to $v$ or $w$ in $G$, then let $i, 1 \leq i \leq 4$, be chosen so that $v_{i}$ is adjacent to $v$ or $w$ in $G$. Taking $v^{*}=v_{i}^{\prime}$, we have that $\left\{v, v^{*}, w\right\}$ dominates $V\left(G_{1}\right)$, a contradiction. Hence, $x$ is adjacent to neither $v$ nor $w$ in $G$. Thus, $N(x) \subset V\left(C_{x}\right)$. Hence, $x$ is adjacent to at least two but at most three vertices on $C_{x}$. If $x$ is adjacent to exactly two vertices on $C_{x}$, then $d_{G}(x)=2$. But then by Subclaim Q1, the set $S$ is not a packing. Hence, $x$ is adjacent to exactly three vertices on $C_{x}$. Without loss of generality, we may assume that $N(x)=\left\{v_{1}, v_{2}, v_{3}\right\}$. Thus by Subclaim Q1, $v_{4}$ is a degree-2 vertex in $G$, i.e., $N\left(v_{4}\right)=\left\{v_{1}, v_{3}\right\}$. Since $v_{2}$ is not an $X$-cut-vertex of $G$, at least one of $v_{1}$ and $v_{3}$ must be adjacent to $v$ or $w$ in $G$. We may assume that $v_{1}$ is adjacent to $v$ or $w$ in $G$. Taking $v^{*}=v_{1}^{\prime}\left(=v_{3}\right)$, we have that $\left\{v, v^{*}, w\right\}$ dominates $V\left(G_{1}\right)$, a contradiction.

We now return to the proof of Claim Q. By Subclaim Q1, $C_{x}$ contains a degree-2 vertex in $G$. We may assume that $d_{G}\left(v_{1}\right)=2$. Since $S$ is a packing in $G$, each vertex in $\left\{v_{2}, v_{3}, v_{4}\right\}$ has degree at least 3 in $G$. Thus, each vertex in $\left\{v_{2}, v_{3}, v_{4}\right\}$ is dominated by $\{v, w, x\}$. Further at least one vertex in $\left\{v_{2}, v_{3}, v_{4}\right\}$ is adjacent to $v$ or $w$ in $G$, while $x$ is adjacent to at least one vertex in $\left\{v_{2}, v_{3}, v_{4}\right\}$.

Subclaim Q3 $x$ is adjacent to neither $v_{2}$ nor $v_{4}$. 
Proof. Suppose that $x$ is adjacent to $v_{2}$ or $v_{4}$. Without loss of generality, we may assume that $x$ is adjacent with $v_{4}$. Suppose $v_{2}$ is adjacent to $v$ or $w$, say to $v$. Let $G^{*}=G-\left\{u, v_{1}, v_{2}, v_{3}\right\}$ and let $X^{*}=X \cup\left\{v, v_{4}\right\}$. Note that $\left|V\left(G^{*}\right)\right|=|V(G)|-4$ and $\left|X^{*}\right|=|X|+2$. By Subclaim Q2, $d_{G^{*}}(x) \geq 2$. Thus, every vertex in $V\left(G^{*}\right) \backslash X^{*}$ has degree at least 2 in $G^{*}$, except possibly for $w$.

Suppose $d_{G^{*}}(w) \geq 1$. Then $\operatorname{bc}\left(G^{*} ; X^{*}\right)+\operatorname{sc}\left(G^{*} ; X^{*}\right)+\delta_{1}\left(G^{*} ; X^{*}\right) \leq 1$. If $\gamma\left(G^{*} ; X^{*}\right) \leq$ $\psi\left(G^{*} ; X^{*}\right)$, then $\gamma\left(G^{*} ; X^{*}\right) \leq \psi\left(G^{*} ; X^{*}\right) \leq \psi(G ; X)-12 / 8+10 / 8+1 / 4=\psi(G ; X)$. Since every $\gamma\left(G^{*} ; X^{*}\right)$-set is an $X$-DS of $G$, we have that $\gamma(G ; X) \leq \gamma\left(G^{*} ; X^{*}\right) \leq \psi\left(G^{*} ; X^{*}\right) \leq$ $\psi(G ; X)$, as desired. Hence we may assume that $\gamma\left(G^{*} ; X^{*}\right)>\psi\left(G^{*} ; X^{*}\right)$. Thus, $G^{*}$ is disconnected and has a component $G_{w}$ such that $\gamma\left(G_{w} ; X_{w}\right)>\psi\left(G_{w} ; X_{w}\right)$ where $X_{w}=$ $X \cap V\left(G_{w}\right)$. Necessarily, $w \in V\left(G_{w}\right)$ and $\left\{v, v_{4}\right\} \cap V\left(G_{w}\right)=\emptyset$. Applying the inductive hypothesis to $G_{w}$, we have that $G_{w} \in \mathcal{F}$ and $X_{w}=\emptyset$. Let $D_{w}$ be a $\gamma\left(G_{w}-w\right)$-set. By Lemma 4(b) and Lemma 5(a), $\left|D_{w}\right|=\left(\left|V\left(G_{w}\right)\right|-1\right) / 3$. Let $F=G-V\left(G_{w}\right)$ and let $X_{F}=$ $X \cup\{u\}$. Applying the inductive hypothesis to $F$, we have that $\gamma\left(F ; X_{F}\right) \leq \psi\left(F ; X_{F}\right)$ since $F$ is connected and $\left|X_{F}\right| \geq 1$. Since $|V(F)|=|V(G)|-\left|V\left(G_{w}\right)\right|,\left|X_{F}\right|=|X|+1$, and $\mathrm{bc}\left(F ; X_{F}\right)+\operatorname{sc}\left(F ; X_{F}\right)+\delta_{1}\left(F ; X_{F}\right)=0$, we have that $\gamma\left(F ; X_{F}\right) \leq \psi\left(F ; X_{F}\right) \leq$ $\psi(G ; X)-3\left|V\left(G_{w}\right)\right| / 8+5 / 8$. Let $D_{F}$ be an $X_{F}$-DS of $F$. Then, $D_{F} \cup D_{w}$ is an $X$-DS of $G$, and so $\gamma(G ; X) \leq\left|D_{F}\right|+\left|D_{w}\right| \leq\left(\psi(G ; X)-3\left|V\left(G_{w}\right)\right| / 8+5 / 8\right)+\left(\left|V\left(G_{w}\right)\right|-1\right) / 3=$ $\psi(G ; X)+\left(7-\left|V\left(G_{w}\right)\right|\right) / 24 \leq \psi(G ; X)$. Hence if $d_{G^{*}}(w) \geq 1$, then $\gamma(G ; X) \leq \psi(G ; X)$, as desired.

Suppose that $w$ is isolated in $G^{*}$; that is, $N_{G}(w)=\left\{u, v_{2}, v_{3}\right\}$. Replacing $v$ in $X^{*}$ with $w$ (and so, $X^{*}=X \cup\left\{v_{4}, w\right\}$ ), we may in a similar manner assume that $N_{G}(v)=\left\{u, v_{2}, v_{3}\right\}$. In this case, let $G^{\prime \prime}=\left(G-\left\{u, v_{1}, v_{3}, v_{4}, w\right\}\right) \cup\{x v\}$ and let $X^{*}=X \cup\left\{v, v_{2}, x\right\}$. Then, $G^{\prime \prime}$ is connected, $\left|V\left(G^{\prime \prime}\right)\right|=|V(G)|-5$ and $\left|X^{\prime \prime}\right|=|X|+3$. Further, $d_{G^{*}}(z) \geq 2$ for all $z \in V\left(G^{\prime \prime}\right) \backslash X^{\prime \prime}$, and $\mathrm{bc}\left(G^{\prime \prime} ; X^{\prime \prime}\right)+\operatorname{sc}\left(G^{\prime \prime} ; X^{\prime \prime}\right)+\delta_{1}\left(G^{\prime \prime} ; X^{\prime \prime}\right)=0$. By the inductive hypothesis, $\gamma\left(G^{\prime \prime} ; X^{\prime \prime}\right) \leq \psi\left(G^{\prime \prime} ; X^{\prime \prime}\right) \leq \psi(G ; X)-5 *(3 / 8)+3 *(5 / 8)=\psi(G ; X)$. Since every $\gamma\left(G^{\prime \prime} ; X^{\prime \prime}\right)$-set is an $X$-DS of $G$, we have that $\gamma(G ; X) \leq \gamma\left(G^{\prime \prime} ; X^{\prime \prime}\right) \leq \psi\left(G^{\prime \prime} ; X^{\prime \prime}\right) \leq$ $\psi(G ; X)$, as desired. We have therefore shown that if $v_{2}$ is adjacent to $v$ or $w$, then $\gamma(G ; X) \leq \psi(G ; X)$, as desired. Hence we may assume that $v_{2}$ is adjacent to neither $v$ nor $w$. Thus, $N_{G}\left(v_{2}\right)=\left\{v_{1}, v_{3}, x\right\}$. Similarly, we may assume that $N_{G}\left(v_{4}\right)=\left\{v_{1}, v_{3}, x\right\}$.

We now consider the graph $F=\left(G-\left\{v_{1}, v_{2}, v_{4}\right\}\right) \cup\left\{x v_{3}\right\}$ and let $X_{F}=X$. Then, $F$ is a connected graph. Note that $|V(F)|=|V(G)|-3$ and that $F$ is a type-2 $G$-reducible graph. As shown in Lemma $2, \gamma(G ; X) \leq \gamma\left(F ; X_{F}\right)+1$. Suppose $F \in \mathcal{F}$ and $X_{F}=\emptyset$. By Lemma 4(b), $\gamma\left(F ; X_{F}\right)=\gamma(F)=(|V(F)|+2) / 3=(|V(G)|-1) / 3$. If $F \notin \mathcal{F}_{13}$, then $G \in \mathcal{F}$, as desired. If $F \in \mathcal{F}_{13}$, then $|V(G)|=16$ and $\gamma(G ; X) \leq \gamma\left(F ; X_{F}\right)+1=6=$ $3|V(G)| / 8=\psi(G ; X)$, as desired. Hence we may assume that if $F \in \mathcal{F}$, then $\left|X_{F}\right| \geq 1$.

Applying the inductive hypothesis to $F$, we have $\gamma\left(F ; X_{F}\right) \leq \psi\left(F ; X_{F}\right)$. Note that $d_{F}(z) \geq 2$ for all $z \in V(F) \backslash X_{F}$, and so $\delta_{1}\left(F ; X_{F}\right)=0$. Further, $\left|X_{F}\right|=|X|$ and $\mathrm{bc}\left(F ; X_{F}\right)+\operatorname{sc}\left(F ; X_{F}\right) \leq 1$. Hence, $\psi\left(F ; X_{F}\right) \leq \psi(G ; X)-9 / 8+1 / 8=\psi(G ; X)-1$. Hence, $\gamma(G ; X) \leq \gamma\left(F ; X_{F}\right)+1 \leq \psi\left(F ; X_{F}\right)+1 \leq \psi(G ; X)$. Hence we have shown that if $x$ is adjacent to $v_{2}$ or $v_{4}$, then $\gamma(G ; X) \leq \psi(G ; X)$, as desired.

By Subclaim Q3, $N(x) \cap V\left(C_{x}\right)=\left\{v_{3}\right\}$. Recall that $v_{1}$ is a degree-2 vertex of $G$. Hence since $S$ is a packing in $G$, each of $v_{2}$ and $v_{4}$ is adjacent to $v$ or $w$. 
Subclaim Q4 Each of $v$ and $w$ is adjacent to exactly one of $v_{2}$ and $v_{4}$.

Proof. Suppose that $v$ is adjacent to both $v_{2}$ and $v_{4}$. Let $G^{\prime \prime}$ be the graph obtained from $G$ by deleting the edges $v_{2} w$ and $v_{4} w$ if these edges are present in $G$, and let $X^{\prime \prime}=X$. Possibly, $G^{\prime \prime}=G$. Then, $\gamma(G ; X) \leq \gamma\left(G^{\prime \prime} ; X^{\prime \prime}\right)$. Let $G^{*}=\left(G-\left\{v_{1}, v_{2}, v_{4}\right\}\right) \cup\left\{v v_{3}\right\}$ and $X^{*}=X$. Then, $\left|V\left(G^{*}\right)\right|=|V(G)|-3$ and $G^{*}$ is a type-2 $G^{\prime \prime}$-reducible graph. As shown in Lemma $2, \gamma\left(G^{\prime \prime} ; X^{\prime \prime}\right) \leq \gamma\left(G^{*} ; X^{*}\right)+1$.

Suppose $G^{*} \in \mathcal{F}$ and $X^{*}=\emptyset$. By Lemma 4(b), $\gamma\left(G^{*} ; X^{*}\right)=\gamma\left(G^{*}\right)=\left(\left|V\left(G^{*}\right)\right|+\right.$ $2) / 3=(|V(G)|-1) / 3$. If $G^{*} \notin \mathcal{F}_{13}$, then $G^{\prime \prime} \in \mathcal{F}$. By Lemma $2, \gamma\left(G^{\prime \prime}\right)=\gamma\left(G^{*}\right)+1=$ $(|V(G)|-1) / 3+1$. If we now restore the graph $G$ from $G^{\prime \prime}$ by adding the deleted edges incident with $w$, if any, we have that either $G \in \mathcal{F}$ or, by repeated applications of Lemma $5(\mathrm{~d})$, we have that $\gamma(G) \leq \gamma\left(G^{\prime \prime}\right)-1=(|V(G)|-1) / 3<3|V(G)| / 8$, as desired. If $G^{*} \in \mathcal{F}_{13}$, then $|V(G)|=16$ and $\gamma(G) \leq \gamma\left(G^{\prime \prime}\right)=\gamma\left(G^{*}\right)+1=6=3|V(G)| / 8=\psi(G ; X)$, as desired. Hence we may assume that if $G^{*} \in \mathcal{F}$, then $\left|X^{*}\right| \geq 1$. Applying the inductive hypothesis to $G^{*}$, we therefore have that $\gamma\left(G^{*} ; X^{*}\right) \leq \psi\left(G^{*} ; X^{*}\right)$. Note that every vertex in $G^{*}$ has degree at least 2 , except possibly for the vertex $w$. Hence, $\delta_{1}\left(G^{*} ; X^{*}\right) \leq 1$.

Suppose that $\delta_{1}\left(G^{*} ; X^{*}\right)=1$. Then, $d_{G^{*}}(w)=1$ and $N_{G}(w)=\left\{u, v_{2}, v_{4}\right\}$. Interchanging the roles of $v$ and $w$, if necessary, we may assume that $N_{G}(v)=\left\{u, v_{2}, v_{4}\right\}$. Hence the graph $M_{G}$ shown in Figure 10 is a subgraph of $G$, where the degrees of the vertices in $V\left(M_{G}\right) \backslash\{x\}$ are unchanged in $G$. We now consider the graph $G_{x}=$ $G-\left\{u, v, v_{1}, v_{2}, v_{3}, v_{4}, w\right\}$ and let $X_{x}=X \cup\{x\}$. Then, $G_{x}$ is a connected graph and every vertex in $V\left(G_{x}\right) \backslash X_{x}$ has degree at least 2 in $G_{x}$. Applying the inductive hypothesis to $G_{x}$, we have that $\gamma\left(G_{x} ; X_{x}\right) \leq \psi\left(G_{x} ; X_{x}\right)$. Since $\left|V\left(G_{x}\right)\right|=|V(G)|-7$, $\left|X_{x}\right|=|X|+1$, and $\mathrm{bc}\left(G_{x} ; X_{x}\right)+\operatorname{sc}\left(G_{x} ; X_{x}\right)+\delta_{1}\left(G_{x} ; X_{x}\right)=0$, we have that $\psi\left(G_{x} ; X_{x}\right) \leq$ $\psi(G ; X)-21 / 8+5 / 8=\psi(G ; X)-2$. Every $X_{x}$-DS of $G_{x}$ can be extended to an $X$-DS of $G$ by adding to it two vertices (for example, we can add $v_{2}$ and $w$ or we can add $v$ and $\left.v_{4}\right)$. Thus, $\gamma(G ; X) \leq \gamma\left(G_{x} ; X_{x}\right)+2 \leq(\psi(G ; X)-2)+2=\psi(G ; X)$, as desired. Hence we may assume that $\delta_{1}\left(G^{*} ; X^{*}\right)=0$.

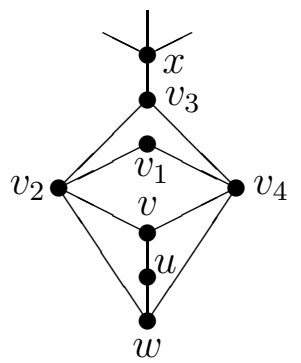

Figure 10: A subgraph $M_{G}$ of $G$.

Since $\left|V\left(G^{*}\right)\right|=|V(G)|-3,\left|X^{*}\right|=|X|, \operatorname{bc}\left(G^{*} ; X^{*}\right)+\operatorname{sc}\left(G^{*} ; X^{*}\right) \leq 1$, and $\delta_{1}\left(G^{*} ; X^{*}\right)=$ 0 , we have that $\psi\left(G^{*} ; X^{*}\right) \leq \psi(G ; X)-9 / 8+1 / 8=\psi(G ; X)-1$. Hence, $\gamma(G ; X) \leq$ $\gamma\left(G^{\prime \prime} ; X^{\prime \prime}\right) \leq \gamma\left(G^{*} ; X^{*}\right)+1 \leq \psi\left(G^{*} ; X^{*}\right)+1 \leq \psi(G ; X)$, as desired.

By Subclaim Q4, we have that $G\left[\left\{v, v_{2}, v_{4}, w\right\}\right]=2 K_{2}$. We may assume $v$ is adjacent with $v_{2}$ and that $w$ is adjacent with $v_{4}$; that is, $N\left(v_{2}\right)=\left\{v, v_{1}, v_{3}\right\}$ and $N\left(v_{4}\right)=\left\{v_{1}, v_{3}, w\right\}$. 
Thus, $\left|\left(N\left(v_{2}\right) \cup N\left(v_{4}\right)\right) \backslash\left\{v_{1}\right\}\right|=3$. Further, $\left\{v_{2}, v_{4}\right\} \subseteq N(v) \cup N(w)$. Hence we have established that the graph $L_{G}$ shown in Figure 11 is a subgraph of $G$, where the edges $v v_{3}$ and $w v_{3}$ may or may not be present in $L_{G}$ and where the degrees of the vertices $u$, $v_{1}, v_{2}$ and $v_{4}$ are unchanged in $G$. Further, $V\left(L_{G}\right) \cap X=\emptyset$ and $x$ is adjacent in $G$ to at least one vertex in $V(G) \backslash V\left(L_{G}\right)$.

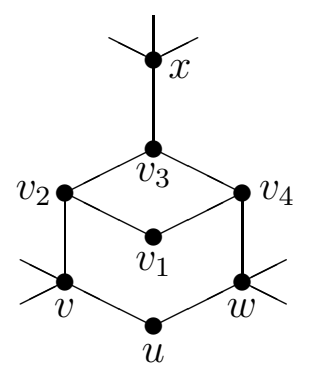

Figure 11: A subgraph $L_{G}$ of $G$.

However if we had considered the degree- 2 vertex $v_{1}$ instead of the degree-2 vertex $u$ in all our computations, we may analogously assume that $d_{G}(v)=3=d_{G}(w)$ and that $v$ and $w$ have a common neighbor $z$ in $G$ that is different from the degree-2 vertex $u$.

Subclaim Q5 $z \neq v_{3}$.

Proof. Suppose that $z=v_{3}$. Then, $N(v)=\left\{u, v_{2}, v_{3}\right\}$ and $N(w)=\left\{u, v_{4}, v_{3}\right\}$. Let $G^{*}=G-\left\{u, v_{1}, v_{2}, w\right\}$ and let $X^{*}=X \cup\left\{v, v_{4}\right\}$. Then, $\left|V\left(G^{*}\right)\right|=|V(G)|-4$ and $\left|X^{*}\right|=$ $|X|+2$. Applying the inductive hypothesis to $G^{*}$, we have that $\gamma\left(G^{*} ; X^{*}\right) \leq \psi\left(G^{*} ; X^{*}\right)$. Every vertex in $V\left(G^{*}\right) \backslash X^{*}$ has degree at least 2 in $G^{*}$, and so $\delta_{1}\left(G^{*} ; X^{*}\right)=0$. Since $\mathrm{bc}(G ; X)+\operatorname{sc}(G ; X)=0$, we have that $\mathrm{bc}\left(G^{*} ; X^{*}\right)+\operatorname{sc}\left(G^{*} ; X^{*}\right)=0$. Thus, $\psi\left(G^{*} ; X^{*}\right)=$ $\psi(G ; X)-12 / 8+10 / 8<\psi(G ; X)$. Since every $\gamma\left(G^{*} ; X^{*}\right)$-DS of $G^{*}$ is a $X$-DS of $G$, we have that $\gamma(G ; X) \leq \gamma\left(G^{*} ; X^{*}\right)<\psi(G ; X)$, as desired.

By Subclaim Q5, $z \neq v_{3}$. Thus, $d_{G}\left(v_{3}\right)=3$ and, analogously, $d_{G}(z)=3$ (by considering the degree-2 vertex $v_{1}$ instead of the degree- 2 vertex $u$ ). If $z=x$, then $|V(G)|=8$ and $X=\emptyset$. Further, $\left\{v, v_{4}\right\}$ is a dominating set of $G$, and so $\gamma(G ; X)=\gamma(G)=2<3=$ $\psi(G ; X)$, as desired. Hence we may assume that $z \neq x$. Let $y$ be the neighbor of $z$ different from $v$ and $w$. Recall that neither $v_{3}$ nor $x$ belongs to $X$. Analogously, we may assume that neither $y$ nor $z$ belongs to $X$. Hence we have established that the graph $R_{G}$ shown in Figure 12 is a subgraph of $G$, where the degrees of the vertices in $V\left(R_{G}\right) \backslash\{x, y\}$ are unchanged in $G$. Further, $V\left(R_{G}\right) \cap X=\emptyset$.

Let $G^{*}=G-\left\{u, v, v_{1}, v_{2}, w, z\right\}$ and $X^{*}=X \cup\left\{v_{4}, y\right\}$. Applying the inductive hypothesis to $G^{*}$, we have that $\gamma\left(G^{*} ; X^{*}\right) \leq \psi\left(G^{*} ; X^{*}\right)$. Note that $\left|V\left(G^{*}\right)\right|=|V(G)|-6$ and $\left|X^{*}\right|=|X|+2$. Every vertex in $V\left(G^{*}\right) \backslash X^{*}$ has degree at least 2 in $G^{*}$, and so $\delta_{1}\left(G^{*} ; X^{*}\right)=0$. Since bc $(G ; X)+\operatorname{sc}(G ; X)=0$, we have that bc $\left(G^{*} ; X^{*}\right)+\operatorname{sc}\left(G^{*} ; X^{*}\right)=0$. Thus, $\psi\left(G^{*} ; X^{*}\right)=\psi(G ; X)-18 / 8+10 / 8=\psi(G ; X)-1$. Every $\gamma\left(G^{*} ; X^{*}\right)$-DS can be extended to a $X$-DS of $G$ by adding to it the vertex $v$, and so $\gamma(G ; X) \leq \gamma\left(G^{*} ; X^{*}\right)+1 \leq$ $\psi(G ; X)$, as desired. This completes the proof of Claim Q. 


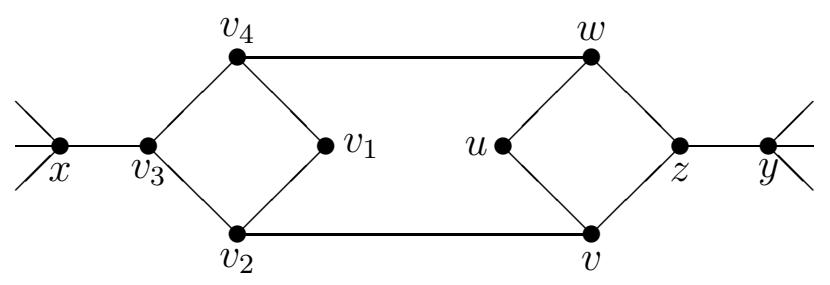

Figure 12: A subgraph $R_{G}$ of $G$.

We now return to the proof of Theorem 4. Recall that $G^{\prime}=G-N[u]$ and that $X^{\prime}=X$. Thus, $\left|V\left(G^{\prime}\right)\right|=|V(G)|-3$ and $\left|X^{\prime}\right|=|X|$. By Claim $\mathrm{O}, \gamma\left(G^{\prime} ; X^{\prime}\right) \leq \psi\left(G^{\prime} ; X^{\prime}\right)$. By Claim P, sc $\left(G^{\prime} ; X^{\prime}\right)=0$. By Claim $\mathrm{Q}, \mathrm{bc}\left(G^{\prime} ; X^{\prime}\right)=0$. Since every vertex at distance 2 from $u$ in $G$ that does not belong to $X$ has degree at least 3 in $G$, we have that $d_{G^{\prime}}(x) \geq 1$ for all $x \in V\left(G^{\prime}\right) \backslash X^{\prime}$.

Claim R $\delta_{1}\left(G^{\prime} ; X^{\prime}\right)=0$.

Proof. Suppose that $\delta_{1}\left(G^{\prime} ; X^{\prime}\right) \geq 1$. Let $v^{\prime}$ be a vertex of degree 1 in $G^{\prime}$. Then, $d_{G}\left(v^{\prime}\right)=3$ and $\{v, w\} \subset N_{G}\left(v^{\prime}\right)$. We may assume that $d_{G}(v) \geq d_{G}(w)$. Let $N_{v}=N_{G}(v) \backslash\{u\}$. Then, $\left|N_{v}\right| \geq 2$. Let $G^{\prime \prime}$ be the graph obtained from $G^{\prime}$ by adding all edges from $v^{\prime}$ to vertices in $N_{v} \backslash\left\{v^{\prime}\right\}$ that are not present in $G$. Suppose $d_{G^{\prime}}\left(v^{\prime}\right)=1$. Then, $\left|N_{v}\right|=2$ and the neighbor $w^{\prime}$ of $v^{\prime}$ different from $v$ and $w$ is adjacent to both $v$ and $w$. Further, $N(v)=N(w)=\left\{u, v^{\prime}, w^{\prime}\right\}$. If $w^{\prime} \notin X$, then $w^{\prime}$ is an $X$-cut-vertex of $G$, a contradicting Claim D that $\mathrm{bc}(G ; X)=0$. Hence, $w^{\prime} \in X$. By Claim $\mathrm{F}, w^{\prime}$ is not a cut-vertex, and so $N\left(w^{\prime}\right)=\left\{v, v^{\prime}, w\right\},|V(G)|=5$ and $|X|=1$. Thus, $\gamma(G ; X)=2<15 / 8+5 / 8=\psi(G ; X)$, as desired. Hence we may assume that $d_{G^{\prime \prime}}(x) \geq 2$ for all $x \in V\left(G^{\prime \prime}\right) \backslash X^{\prime}$, and so $\delta_{1}\left(G^{\prime \prime} ; X^{\prime \prime}\right)=0$. Note that bc $\left(G^{\prime \prime} ; X^{\prime \prime}\right)+\operatorname{sc}\left(G^{\prime \prime} ; X^{\prime \prime}\right) \leq \mathrm{bc}\left(G^{\prime} ; X^{\prime}\right)+\operatorname{sc}\left(G^{\prime} ; X^{\prime}\right)+1=1$. Let $G_{v}$ be the component of $G^{\prime \prime}$ containing $v^{\prime}$ and let $X_{v}=X \cap V\left(G_{v}\right)$. If $G^{\prime \prime} \neq G_{v}$, let $G_{w}=G^{\prime \prime}-V\left(G_{v}\right)$ and let $X_{w}=X \cap V\left(G_{w}\right)$. Possibly, $G_{w}$ is disconnected.

Claim R1 If $G^{\prime \prime} \neq G_{v}$, then $\gamma\left(G_{w} ; X_{w}\right) \leq \psi\left(G_{w} ; X_{w}\right)$.

Proof. Suppose $G^{\prime \prime} \neq G_{v}$. By construction, $d_{G_{w}}(x) \geq 2$ for all $x \in V\left(G_{w}\right) \backslash X_{w}$, and so $\delta_{1}\left(G_{w} ; X_{w}\right)=0$. Note that if $K$ is a component of $G_{w}$, then $K$ is also a component of $G^{\prime}$, and so by Claim $\mathrm{N}$ and Claim $\mathrm{O}, \gamma\left(K ; X_{K}\right) \leq \psi\left(K ; X_{K}\right)$ where $X_{K}=X \cap V(K)$. Further, by Claim P and Claim Q, we have that bc $\left(G_{w} ; X_{w}\right)+\operatorname{sc}\left(G_{w} ; X_{w}\right)=\mathrm{bc}\left(G^{\prime} ; X^{\prime}\right)+$ $\operatorname{sc}\left(G^{\prime} ; X^{\prime}\right)=0$. Hence, $\gamma\left(G_{w} ; X_{w}\right) \leq \psi\left(G_{w} ; X_{w}\right)$.

If $G^{\prime \prime} \neq G_{v}$, let $D_{w}$ be a $\gamma\left(G_{w} ; X_{w}\right)$-set. By Claim R1, $\left|D_{w}\right|=\gamma\left(G_{w} ; X_{w}\right) \leq \psi\left(G_{w} ; X_{w}\right)$. Let $G_{u}=G\left[V\left(G_{v}\right) \cup\{u, v, w\}\right]$ and let $X_{u}=X \cap V\left(G_{u}\right)$.

Claim R2 $\gamma\left(G_{u} ; X_{u}\right) \leq \gamma\left(G_{v} ; X_{v}\right)+1$

Proof. Let $D_{v}$ be an $\gamma\left(G_{v} ; X_{v}\right)$-set. If $v^{\prime} \in D_{v}$, let $D=D_{v} \cup\{v\}$. If $v^{\prime} \notin D_{v}$ but $D_{v}$ contains a vertex in $N_{v}$, let $D=D_{v} \cup\{w\}$. If $D_{v}$ contains no vertex in $N_{v}$, let $D=D_{v} \cup\{u\}$. In all three cases, $D$ is an $\gamma\left(G_{u} ; X_{u}\right)$-DS of $G$. Hence, $\gamma\left(G_{u} ; X_{u}\right) \leq$ $\left|D_{v}\right|+1=\gamma\left(G_{v} ; X_{v}\right)+1$. 
Claim R3 $\gamma\left(G_{v} ; X_{v}\right) \leq \psi\left(G_{v} ; X_{v}\right)$.

Proof. Suppose $\gamma\left(G_{v} ; X_{v}\right)>\psi\left(G_{v} ; X_{v}\right)$. Applying the inductive hypothesis to $G_{v}$, we have that $G_{v} \in \mathcal{F}$ and $X_{v}=\emptyset$. Thus, $X=\emptyset$ or, if $G^{\prime \prime} \neq G_{v}$, then $X=X_{w}$ (possibly, $X_{w}=\emptyset$ ). Further, $\gamma\left(G_{v} ; X_{v}\right)=\gamma\left(G_{v}\right)$ and $\gamma\left(G_{u} ; X_{u}\right)=\gamma\left(G_{u}\right)$. By Claim R2, $\gamma\left(G_{u}\right) \leq \gamma\left(G_{v}\right)+1$. By Lemma $4(\mathrm{~b}), \gamma\left(G_{v}\right)=\left(\left|V\left(G_{v}\right)\right|+2\right) / 3$.

If $G_{v} \in \mathcal{F}_{13}$, then $\left|V\left(G_{u}\right)\right|=16$ and $\gamma\left(G_{u}\right) \leq \gamma\left(G_{v}\right)+1=6=3\left|V\left(G_{u}\right)\right| / 8=$ $\psi\left(G_{u} ; X_{u}\right)$. On the one hand, if $G=G_{u}$, then $\gamma(G ; X)=\gamma(G)=\gamma\left(G_{u}\right) \leq \psi\left(G_{u} ; X_{u}\right)=$ $\psi(G ; X)$, as desired. On the other hand, if $G \neq G_{u}$, then $\gamma(G ; X)=\gamma\left(G_{u}\right)+\gamma\left(G_{w} ; X_{w}\right) \leq$ $\psi\left(G_{u} ; X_{u}\right)+\psi\left(G_{w} ; X_{w}\right)=\psi(G ; X)$, as desired. Hence we may assume $G_{v} \in \mathcal{F}_{4} \cup \mathcal{F}_{7} \cup \mathcal{F}_{10}$, and so $\left|V\left(G_{v}\right)\right| \in\{4,7,10\}$. In particular, $\left|V\left(G_{u}\right)\right| \geq 7$.

Let $G_{u}^{\prime}$ be obtained from $G_{u}$ by deleting all edges, if any, in $G_{u}$ that are incident with $w$, except for the two edges $u w$ and $v^{\prime} w$. Note that $v^{\prime} w u v$ is a path in $G_{u}^{\prime}$ and that each of $u$ and $w$ has degree 2 in $G_{u}^{\prime}$. Since $G_{v}$ is a type- $1 G_{u}^{\prime}$-reducible graph and $G_{v} \in \mathcal{F}$, the graph $G_{u}^{\prime} \in \mathcal{F}$. By Lemma $1, \gamma\left(G_{u}^{\prime}\right)=\gamma\left(G_{v}\right)+1=\left(\left|V\left(G_{v}\right)\right|+2\right) / 3+1=\left(\left|V\left(G_{u}\right)\right|-1\right) / 3+1$. If we now restore the graph $G_{u}$ from $G_{u}^{\prime}$ by adding the deleted edges incident with $w$, we have that either $G_{u} \in \mathcal{F}$ or, by repeated applications of Lemma $5(\mathrm{~d})$, we have that $\gamma\left(G_{u}\right) \leq \gamma\left(G_{u}^{\prime}\right)-1=\left(\left|V\left(G_{u}\right)\right|-1\right) / 3<3\left|V\left(G_{u}\right)\right| / 8=\psi\left(G_{u} ; X_{u}\right)$.

If $G=G_{u}$, then either $G \in \mathcal{F}$ or $\gamma(G ; X)=\gamma(G)=\gamma\left(G_{u}\right)<\psi\left(G_{u} ; X_{u}\right)=\psi(G ; X)$, as desired. Further if $G \neq G_{u}$ and $G_{u} \notin \mathcal{F}$, then $\gamma(G ; X) \leq \gamma\left(G_{u}\right)+\gamma\left(G_{w} ; X_{w}\right)<$ $\psi\left(G_{u} ; X_{u}\right)+\psi\left(G_{w} ; X_{w}\right)=\psi(G ; X)$, as desired. Hence we may assume that $G \neq G_{u}$ and $G_{u} \in \mathcal{F}$. Let $D_{u}$ be a $\gamma\left(G_{u}-w\right)$-set. By Lemma $5(\mathrm{a}),\left|D_{u}\right|=\gamma\left(G_{u}-w\right) \leq \gamma\left(G_{u}\right)-1=$ $\left(\left|V\left(G_{u}\right)\right|-1\right) / 3$. We now let $w^{\prime} \in N(w) \backslash V\left(G_{u}\right)$. Applying the inductive hypothesis to $G_{w}$, we have that $\gamma\left(G_{w} ; X_{w} \cup\left\{w^{\prime}\right\}\right) \leq \psi\left(G_{w} ; X_{w} \cup\left\{w^{\prime}\right\}\right)=\psi\left(G_{w} ; X_{w}\right)+5 / 8=$ $\psi(G ; X)-3\left|V\left(G_{u}\right)\right| / 8+5 / 8$. Every $\gamma\left(G_{w} ; X_{w} \cup\left\{w^{\prime}\right\}\right)$-set can be extended to an $X$ DS of $G$ by adding to it the set $D_{u}$. Hence, $\gamma(G ; X) \leq \gamma\left(G_{w} ; X_{w} \cup\left\{w^{\prime}\right\}\right)+\left|D_{u}\right| \leq$ $\left(\psi(G ; X)-3\left|V\left(G_{u}\right)\right| / 8+5 / 8\right)+\left(\left|V\left(G_{u}\right)\right|-1\right) / 3=\psi(G ; X)+\left(7-\left|V\left(G_{u}\right)\right|\right) / 24 \leq \psi(G ; X)$, as desired.

By Claim R1 and Claim R3, we have that $\gamma\left(G^{\prime \prime} ; X^{\prime \prime}\right) \leq \psi\left(G^{\prime \prime} ; X^{\prime \prime}\right)$. As observed earlier, bc $\left(G^{\prime \prime} ; X^{\prime \prime}\right)+\operatorname{sc}\left(G^{\prime \prime} ; X^{\prime \prime}\right) \leq 1$ and $\delta_{1}\left(G^{\prime \prime} ; X^{\prime \prime}\right)=0$. Since $\left|V\left(G^{\prime \prime}\right)\right|=|V(G)|-3$ and $\left|X^{\prime \prime}\right|=|X|$, we have that $\psi\left(G^{\prime \prime} ; X^{\prime \prime}\right) \leq \psi(G ; X)-9 / 8+1 / 8=\psi(G ; X)-1$. Every $\gamma\left(G^{\prime \prime} ; X^{\prime \prime}\right)$-set can be extended to a $X$-DS of $G$ by adding to it the vertex $u$, and so $\gamma(G ; X) \leq \gamma\left(G^{\prime \prime} ; X^{\prime \prime}\right)+1 \leq \psi(G ; X)$, as desired. This completes the proof of Claim R.

We return to the proof of Theorem 4 one last time. As established earlier, $\left|V\left(G^{\prime}\right)\right|=$ $|V(G)|-3,\left|X^{\prime}\right|=|X|, \gamma\left(G^{\prime} ; X^{\prime}\right) \leq \psi\left(G^{\prime} ; X^{\prime}\right), \operatorname{sc}\left(G^{\prime} ; X^{\prime}\right)=0$ and $\mathrm{bc}\left(G^{\prime} ; X^{\prime}\right)=0$. By Claim R, $\delta_{1}\left(G^{\prime} ; X^{\prime}\right)=0$. Thus, $\psi\left(G^{\prime} ; X^{\prime}\right)=\psi(G ; X)-9 / 8<\psi(G ; X)-1$. Every $\gamma\left(G^{\prime} ; X^{\prime}\right)$-DS can be extended to a $X$-DS of $G$ by adding to it the vertex $u$, and so $\gamma(G ; X) \leq \gamma\left(G^{\prime} ; X^{\prime}\right)+1<\psi(G ; X)$. This completes the proof of Theorem 4 . 